\title{
How Useful is an Integrated SPECT/CT in Clinical Setting and Research?: Evaluation of a Low Radiation Dose 4 Slice System ${ }^{\S}$
}

\author{
Tarik Belhocine ${ }^{*}$, Irina Rachinsky $^{1}$, Cigdem Akincioglu ${ }^{1}$, Sanjay Gambhir ${ }^{1}$, Brad Wilcox $^{1}$, \\ William Vezina ${ }^{1}$, Larry Stitt ${ }^{2}$, Albert Driedger ${ }^{1}$ and Jean-Luc Urbain ${ }^{1}$
}

\author{
Departments of ${ }^{1}$ Nuclear Medicine and ${ }^{2}$ Epidemiology \& Biostatistics, London Health Sciences Centre, The University \\ of Western Ontario, London, N6A 4G5, Ontario, Canada
}

\begin{abstract}
Hybrid imaging is becoming a popular technology in nuclear medicine. We have evaluated the added value of an integrated SPECT/low-dose multislice CT over conventional planar/SPECT nuclear imaging. Phantom and clinical studies were performed on the Infinia ${ }^{\mathrm{TM}}$ Hawkeye ${ }^{\mathrm{TM}} 4$ slice (HWK-4) with an upgraded software package (Xeleris 2.05v) from GE Heatlthcare to assess 1) the benefit of CT for contrast-resolution, attenuation correction, and anatomic localisation; 2) the impact of hybrid imaging in 456 consecutive patients in a clinical setting. SPECT/CT data were compared to conventional planar/SPECT data and correlated to clinical, biochemical, morphological imaging, angiography, and pathology findings. SPECT/CT was well tolerated by the patients with minimal CT irradiation dose $(<$ $2 \mathrm{mSv}$ ). HWK-4 provided useful attenuation correction for its routine use in MPI and accurate anatomic localisation of physiological and pathological foci in ${ }^{99 \mathrm{~m}} \mathrm{Tc}-\mathrm{RBC},{ }^{99 \mathrm{~m}} \mathrm{Tc}-\mathrm{HMPAO}-\mathrm{WBC},{ }^{131 / 123} \mathrm{I}-\mathrm{MIBG}$, Octreoscan ${ }^{\circledR}$, and ${ }^{67} \mathrm{Ga}$ studies. Low-dose multislice CT also helped detect gross morphological abnormalities. Hybrid imaging had a significant impact in ProstaScint ${ }^{\circledR}$ and parathyroid imaging for image-guided intervention. In bone imaging and differentiated thyroid cancers, SPECT/CT was able to clarify equivocal findings from planar whole-body scan. SPECT/CT was also found useful to precisely localize sentinel lymph nodes. Research protocols are being evaluated for half-time acquisition with resolution recovery and quantification of tracer distribution. SPECT/low-dose multislice CT has been successfully implemented in routine clinical practice. CT provided added value for effective attenuation correction and accurate anatomic localisation of disease with an impact on patient management.
\end{abstract}

Keywords: Hybrid imaging, SPECT/CT, low-dose multislice CT, clinical impact.

\section{INTRODUCTION}

In recent years, hybrid imaging has become a powerful diagnostic tool in nuclear medicine, particularly with current models of positron emission tomography/computed tomography (PET/CT) [1]. Similarly, single photon emission tomography/computed tomography (SPECT/CT) in routine practice is increasingly being accepted as a modality of choice for the assessment of many diseases [2]. The combination of functional and anatomic information in a 'one-stop shop' format has been shown to increase nuclear medicine physicians' confidence for image interpretation, while giving clinicians a more accurate evaluation of disease [3-6]. Although the PET/CT trend is firmly established, SPECT/CT is still undergoing evaluation $[7,8]$. No clear conclusion has been drawn regarding the required CT performance for optimal SPECT/CT. The choice between low-dose and high-dose SPECT/CT remains challenging with the latter providing higher spatial anatomic resolution [9]. As various PET tracers allowing more specific assessment of biological functions are developed, the long-

*Address correspondence to this author at the South Street Hospital, Department of Nuclear Medicine, London Health Sciences Centre 375 South Street London, N6A 4G5, London, Ontario, Canada; Tel: 519-6858500, Ext. 76069; Fax: 519-667-6734; E-mail: tbelhocine@yahoo.ca

${ }^{\S}$ Part of this work was presented as selected poster at the Lawson Research Day (London, Canada, March 2007). term role of SPECT/CT is questioned [10-12]. On the other hand, the requirement of a cyclotron for the synthesis of PET tracers as well as the lack of permisive reimbursement policies for the performance of PET/CT may be problematic, especially for non-oncological applications. In addition, ${ }^{18} \mathrm{~F}$ fluorodeoxyglucose imaging $\left({ }^{18} \mathrm{FDG}\right)$ with PET/CT remains of limited value in a number of routine indications such as sentinel lymph node detection, ${ }^{131} \mathrm{I}$-avid differentiated thyroid cancers, prostate cancer, and well-differentiated neuroendocrine tumors [13-16]. Myocardial perfusion imaging (MPI) is commonly performed with SPECT, and more recently with SPECT/CT $[17,18]$.

In this article, we report our experience with the InfiniaHawkeye 4, an integrated SPECT/CT system in a general hospital. This hybrid camera, which was the first of its kind to be installed in North America, combines a low-dose multislice CT with a dual-head camera in a single-gantry. We analyzed the camera performance and the results obtained in clinical practice. We also report on the research potential offered by this model of SPECT/low-dose multislice CT.

\section{MATERIAL AND METHODOLOGY}

\section{Study Design}

Our project evaluated a new SPECT/CT device before its commercialisation. The objectives were 1) to assess the benefit of the equipment, gained by the shortened CT time 
and improved CT image quality relative to the previous single slice $\mathrm{CT}, 2$ ) to evaluate existing and new hybrid protocols workflow, 3) to refine CT acquisition and reconstruction parameters, 4) to evaluate image registration package and 5) to evaluate the product usability and quality. This project was approved by The University of Western Ontario Ethics Review Board.

\section{Hardware}

The Infinia-Hawkeye 4 (HWK-4) was the SPECT/CT model used in this project. This hybrid device incorporates a low-dose CT (current $=1.0$ to $2.5 \mathrm{~mA}$, voltage $=120$ or 140 $\mathrm{kV}, 512 \times 512$ matrix, Effective dose $<2 \mathrm{mSv}$ ) and a dualhead gamma camera (59 photomultipliers/head, $3 / 8$ inch NaI crystal) in a single gantry. The HWK-4 provides a $20 \mathrm{~mm}$ axial coverage with a $5 \mathrm{~mm}$ thickness per slice. CT data were acquired in axial mode for brain studies or in helical mode for cardiac and non-cardiac studies. The axial field of view (FOV) covered by the SPECT/CT is $40 \mathrm{~cm}$. Based on the FOV scanned, either a partial scan or a full scan, the CT duration was about 2 min and $4 \mathrm{~min}$ for cardiac and noncardiac studies, respectively.

\section{Software}

Xeleris version 2.05 was used for data processing and analysis. This workstation was upgraded to allow reconstruction and interpretation of SPECT/CT data with dedicated software applications, particularly for bone SPECT/CT studies (Whole-body slicing for Infinia, Wholebody Evolution), cardiac SPECT/CT studies (Myometrix, Myovation), and oncology studies (Volumetrix for Hawkeye Oncology).

\section{Phantom Studies}

Attenuation correction (AC) on the Infinia-Hawkeye 4 was assessed with an anthropomorphic-body phantom (Data Spectrum Corp.). For non cardiac SPECT/CT, various radioactive sources $\left({ }^{131} \mathrm{I},{ }^{99 \mathrm{~m}} \mathrm{Tc},{ }^{67} \mathrm{Ga}\right.$, and $\left.{ }^{111} \mathrm{In}\right)$ were inserted at the center and at the periphery of the habitus to simulate deep and superficial foci in a clinical SPECT/CT acquisition (i.e. ${ }^{67} \mathrm{Ga}$ study, ${ }^{99 \mathrm{~m}} \mathrm{Tc}-\mathrm{MDP}$ bone scan, ${ }^{11}$ In-Octreoscan, and $\left.{ }^{13} \mathrm{I}-\mathrm{MIBG}\right)$. For cardiac SPECT/ CT, experiments were performed to assess the effectiveness of iterative reconstruction with and without attenuation correction (IRAC vs IRNC) in normal and abnormal conditions. These included a reference acquisition with the cardiac insert in air (no attenuation). Various SPECT/CT acquisitions were also performed in simulated clinical conditions with variation of CT parameters $(2.5 \mathrm{~mA}$, $1.0 \mathrm{~mA}, 140 \mathrm{kV}, 120 \mathrm{kV}$, axial mode, helical mode, soft tissue filter, 256x256 matrix, breast attenuation, liver attenuation, extra-cardiac activity, right truncation, left truncation, misalignment, oral contrast, i.v contrast, transmural and non-transmural defects) using an anthropomorphic phantom with a cardiac insert.

\section{Clinical Studies}

From April 2006 to December 2007, 456 consecutive patients underwent SPECT/CT studies for various clinical indications (Table 1). These applications included myocardial perfusion imaging (MPI) studies $(n=70)$, bone scans $(n=51)$, sentinel lymph node detection $(n=12),{ }^{99 m}$ Tc-
MIBI parathyroid studies ( $\mathrm{n}=42),{ }^{131} \mathrm{I}$ scans for differentiated thyroid cancers $(n=42)$, ProstaScint ${ }^{\circledR}$ studies $(n=27),{ }^{67} \mathrm{Ga}$ studies $(\mathrm{n}=40),{ }^{123} \mathrm{I}$-MIBG $(\mathrm{n}=6),{ }^{131} \mathrm{I}$-MIBG $(\mathrm{n}=13),{ }^{99 \mathrm{~m}} \mathrm{Tc}-$ MIBI and ${ }^{201} \mathrm{Tl}$ brain studies $(\mathrm{n}=7)$, Octreoscan ${ }^{\circledR}$ studies $(\mathrm{n}=51),{ }^{99 \mathrm{~m}}$ Tc-labelled red blood cell studies $(\mathrm{n}=11)$, and ${ }^{99 \mathrm{~m}} \mathrm{Tc}$-HMPAO-labelled white blood cell studies $(\mathrm{n}=38)$. In clinical research, a number of patients were evaluated for pre-treatment dosimetry calculations, particularly for benign thyroid diseases such as multinodular goiter $(n=3)$ or Graves' disease $(n=1)$. Patients were also imaged following radioimmunotherapy (RIT) with $\operatorname{Bexxar}^{\circledR}(n=4)$ or chemoembolisation using Lipiocis $^{\circledR} \quad(n=5)$ to document the distribution of therapeutic radiation dose. Additionally, a half-time resolution recovery (HTRR) package for SPECT/low-dose multislice CT MPI was assessed in 33 cardiology patients.

Table 1. Clinical Indications Evaluated on the Infinia ${ }^{\mathrm{TM}}$ Hawkeye $^{\mathrm{TM}} 4$ Slice (GE Healthcare)

\begin{tabular}{|l|c|}
\hline \multicolumn{1}{|c|}{ Clinical Indications } & Number of Patients \\
\hline \hline${ }^{99 m}$ Tc-MIBI MPI & 70 \\
\hline${ }^{131}$ I scans (DTC) & 42 \\
\hline Parathyroid imaging & 42 \\
\hline SLN detection & 12 \\
\hline Bone imaging & 51 \\
\hline ProstaScint ${ }^{\circledR}$ imaging & 27 \\
\hline${ }^{99 m}$ Tc-RBC imaging & 11 \\
\hline${ }^{99 m}$ Tc-HMPAO-WBC & 38 \\
\hline${ }^{67}$ Ga imaging & 40 \\
\hline${ }^{131 / 123}$ I-MIBG imaging & 19 \\
\hline Octreoscan ${ }^{\circledR}$ imaging & 51 \\
\hline Brain imaging & 7 \\
\hline${ }^{131}$ I Pre-therapy (BTD) & 4 \\
\hline${ }^{131}$ I-Bexar post-therapy & 4 \\
\hline${ }^{131}$ I-Lipiodol post-therapy & 5 \\
\hline${ }^{99 m}$ Tc-MIBI MPI with HTRR & 33 \\
\hline & $\mathbf{4 5 6}$ \\
\hline
\end{tabular}

MPI, myocardial perfusion imaging; DTC, differentiated thyroid cancer; SLN, sentinel lymph node; RBC, red blood cell; WBC, white blood cell; BTD, benign thyroid diseases; HTRR, half-time scan with resolution recovery.

\section{ANALYSIS}

In phantom studies, the effect of attenuation correction (AC) in non cardiac applications was assessed on the Volumetrix for Oncology application by means of regions of interest (ROIs) manually defined in centrally and peripherally located radioactive sources $\left({ }^{131} \mathrm{I},{ }^{67} \mathrm{Ga},{ }^{111} \mathrm{In}\right.$, and ${ }^{99 \mathrm{~m}} \mathrm{Tc}$ ). The effectiveness of attenuation correction in MPI was evaluated according to the O'Connor methodology [19]. A 20-segment analysis was also performed on the $Q G S / Q P S / M y o m e t r i x$ application from the Xeleris $2.05 \mathrm{v}$ for $2.5 \mathrm{~mA}$ and $1.0 \mathrm{~mA}$, respectively. For evaluation of counts recovery per segment after $\mathrm{AC}$, an acquisition with the cardiac insert in air (i.e. no attenuation) was used as gold 
standard. In clinical studies, we evaluated patient tolerability for hybrid acquisitions, CT performance for anatomy and attenuation correction, and SPECT/CT added-value in routine practice and research.

It was possible to correlate some SPECT/CT studies with a pathology gold standard after surgery. These indications included parathyroid imaging and sentinel lymph node detection. In DTC patients, the SPECT/CT scans were compared to planar ${ }^{131}$ I-whole body scan $\left({ }^{131} \mathrm{I}\right.$-WBS $)$ and related to Thyrogen ${ }^{\circledR}$-stimulated $\mathrm{Tg}$ levels, and morphological imaging such as neck ultrasound, diagnostic $\mathrm{CT}$, and brain MRI. In prostate cancer patients evaluated by ProstaScint ${ }^{\circledR}$, SPECT/CT findings were compared to planar whole-body (PWB) and SPECT data, respectively. Final results were associated with the Gleason score, PSA levels, CT/MRI, and bone scan. All pathology, clinical, biochemical, and imaging data were reviewed by a multidisciplinary prostate cancer team. In patients with neuroendocrine tumors, SPECT/CT studies performed for ${ }^{131 / 123}$ I-MIBG and/or ${ }^{111}$ In-Octreotide (Octreoscan ${ }^{\circledR}$ ) were compared with PWB scans for detection and localisation of additional foci. Similarly, ${ }^{67} \mathrm{Ga}$ SPECT/CT was compared to planar WBS in patients evaluated for lymphoma or suspicion of inflammation or infection. SPECT/CT results were associated with morphological imaging (CT/MRI) and histopathological findings. We also evaluated the usefulness of bone SPECT/CT in patients with equivocal planar WB scans including 34 patients referred for initial staging or restaging of cancer, and 17 patients with no cancer at the time of the scan referred for lower back pain, neck pain, chest pain, joint pain, and osteomyelitis. Bone SPECT/CT studies were performed with a new application Bone Evolution, a half-time acquisition protocol (16sec/proj) with an iterative reconstruction allowing collimator-detector response compensation for resolution recovery; Bone Evolution SPECT/CT findings were compared to CT/MRI studies. In patients with a suspicion of osteomyelitis or active Crohn's disease, ${ }^{99 \mathrm{~m}} \mathrm{Tc}$-HMPAO-WBC imaging was performed with SPECT/CT and compared to planar imaging. In patients imaged for a suspicion of liver and/or splenic hemangiomas or splenosis, ${ }^{99 \mathrm{~m}} \mathrm{Tc}$-red blood cell imaging (RBC) was also performed with a SPECT/low-dose multislice CT. Imaging results were associated with clinical and biological data, morphological imaging, surgical and pathology results.

In cardiology patients, SPECT/CT MPI images were evaluated before and after attenuation correction (IRNC vs IRAC) by using a 5-point scale analysis (definitely abnormal, probably abnormal, equivocal, probably normal, and definitely normal) with knowledge of clinical data. Final results were obtained in consensus and compared to angiography. Bayesian analysis was performed in normal studies.

In clinical research, 13 oncology patients were evaluated using SPECT/CT in addition to conventional planar imaging before or after internal radiation therapy (IRT); in 33 cardiology patients, we evaluated the feasibility of half-time MPI with attenuation correction, scatter correction, and collimator-detector response compensation for resolution recovery $(7.5 \mathrm{~min}$ SPECT $+2.5 \mathrm{~min} \mathrm{CT})$ versus full-time MPI (15min SPECT $+2.5 \mathrm{~min} \mathrm{CT})$ in terms of image quality and diagnostic accuracy. In this preliminary evaluation, all half-time SPECT/CT studies were reprocessed by GE application managers using Poisson resampling of the raw data in order to derive a dataset simulating an acquisition with 50\% the scan time (for high dose stress) and $60 \%$ scan time (for low dose rest) as in the original data.

\section{STATISTICS}

In non cardiac phantom studies, maximum counts were calculated on the Xeleris 2.05v (Volumetrix for Oncology) for all manual ROIs before and after attenuation correction (IRNC vs IRAC). In cardiac phantom studies, the apex-tobase uniformity, the maximum slice uniformity, and the global uniformity were calculated for various CT acquisitions [19]. A 20-segment polar maps analysis was also performed on the Xeleris 2.05v (QGS/QPS/Myometrix) for a routine protocol $(2.5 \mathrm{~mA}, 140 \mathrm{kVp})$ and an optimized protocol $(1.0 \mathrm{~mA}, 140 \mathrm{kVp})$. IRAC and IRNC data were compared using both the paired Student t-test, assuming an underlying normal distribution, and the Wilcoxon signed rank test, allowing for deviations from normality.

In clinical studies, the sensitivity, the specificity, and the diagnostic accuracy were calculated according to the common definitions. Comparisons between planar/SPECT imaging and SPECT/CT diagnostic performances were performed using the Fisher's exact tests.

A p-value $<0.05$ was considered as significant. SAS 9.1 software package (Institute Inc, Cary, NC) was used for statistical analyses.

\section{RESULTS}

\section{Patient Tolerability}

SPECT/CT was well tolerated by patients in routine clinical practice. The SPECT/CT was included in the patients' work-flow with no particular preparation. In 2 claustrophobic patients $(<0.1 \%)$, the acquisition was stopped before completion. Many patients, especially obese subjects, experienced transient shoulder pain due to the positioning of the arms up. In studies with a relatively short acquisition time (<30 min), SPECT was usually acquired first (MPI, SLN detection, Bone scan). In studies with acquisitions longer than $30 \mathrm{~min}$ (i.e. ${ }^{67} \mathrm{Ga}$, ProstaScint ${ }^{\circledR}$, ${ }^{131 / 123} \mathrm{I}$-MIBG, Octreoscan $\left.{ }^{\circledR}\right)$, CT was acquired first for the patients' convenience.

\section{Phantom Studies}

For non-cardiac SPECT/CT studies, the CT-based AC from HWK-4 increased the maximum counts statistics in peripherally or centrally located radioactive sources by a factor 3.4 for ${ }^{131} \mathrm{I},{ }^{111} \mathrm{In}$, and ${ }^{67} \mathrm{Ga}$ to 5.9 for ${ }^{99 \mathrm{~m}} \mathrm{Tc}$, when IRAC images were compared to IRNC images. These results predict the benefits expected from $\mathrm{AC}$ in clinical setting. They also indicate the need for an appropriate re-scaling of tracer uptakes for accurate interpretation, eventually by means of fully- or semi-quantitative scores.

Cardiac phantom studies demonstrated the effectiveness of CT-based AC when applied in cases of breast attenuation and liver attenuation, especially for a clinical routine 


\section{Apex-to-Base Uniformity : IRAC vs. IRNC}

\section{$\%$ Uniformity}

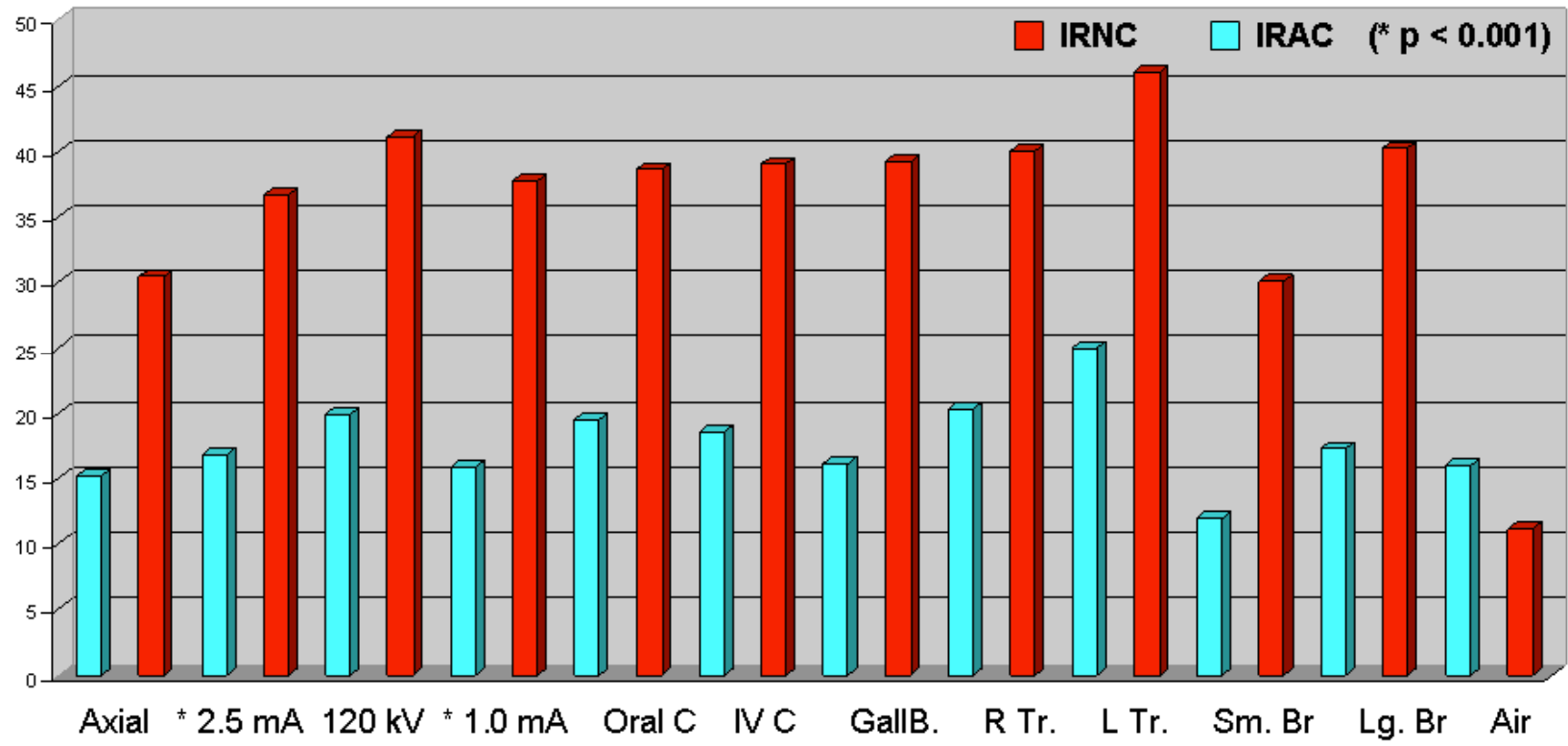

\section{CT Aquisitions}

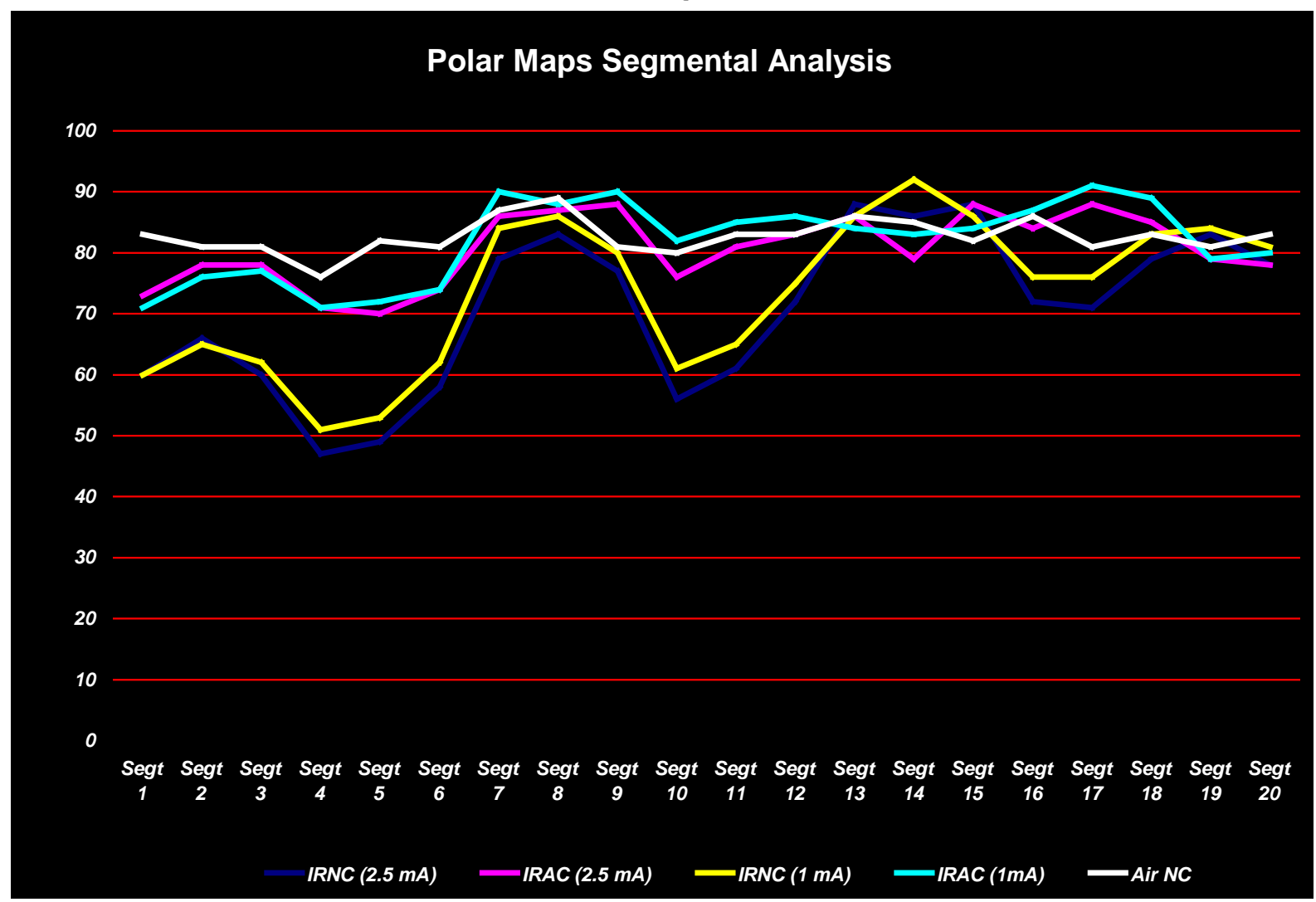

Fig. (1). Cardiac phantom studies were performed on the Infinia-Hawkeye 4 (GE Healthcare) to assess the effectiveness of CT-based attenuation correction (AC). The acquisition with no attenuation (cardiac insert in air) was used as gold standard. According to the O'Connor methodology [19], IRAC allowed a significant improvement of the apex-to-base uniformity $(\mathrm{p}<0.001)$ in the left myocardium ventricle compared to IRNC (top). Similarly, a segmental analysis (bottom) showed the significant effect of AC for recovery of loss counts for both $2.5 \mathrm{~mA}$ (routine protocol) and $1.0 \mathrm{~mA}$ (optimized protocol). Abbreviations: Oral C, oral contrast; IV C, intravenous contrast; GallB; gallbladder hot-spot; L Tr, left truncation; R Tr, right truncation; Sm Br, small breast; Lg Br, large breast. 
acquisition with a $2.5 \mathrm{~mA}$ (Fig. 1, top). They also identified the potential utility of $1.0 \mathrm{~mA}$ acquisition for maintaining an effective AC, while reducing the weighted CT Dose Index (1.6 mGy) compared to $2.5 \mathrm{~mA}$ (4.02 mGy). Variations in the slice uniformity was significantly reduced $(<20 \%)$ after IRAC $v s$ IRNC for all clinically simulated conditions, especially for the apex-to-base uniformity in liver attenuation with both 2.5 $\mathrm{mA}(16.7 \%$ vs $36.7 \%$; $\mathrm{p}<0.001)$ and $1.0 \mathrm{~mA}(15.4 \%$ vs $37.4 \%$; $\mathrm{p}<0.001)$. On polar maps, a significant difference was found between IRNC and IRAC normalised scores per segment $(p<0.001)$. A significant difference $(p=0.001)$ was found between IRNC and non attenuated data (i.e. cardiac insert in air), while no statistical difference was observed for both $2.5 \mathrm{~mA}(\mathrm{p}=0.077)$ and $1.0 \mathrm{~mA}(\mathrm{p}=0.563)$ between IRAC and non attenuated data (i.e. cardiac insert in air). Segmental analysis (Fig. 1, bottom) showed the effect of AC for a significant recovery of loss counts for $2.5 \mathrm{~mA}$ (routine protocol) and $1.0 \mathrm{~mA}$ (optimized protocol), particularly in the basal inferior segment (segment 4), the mid inferior segment (segment 10), and the apical inferior segment (segment 16).

\section{CT Performance}

The use of concomitant low-dose multislice CT with SPECT allowed simultaneous anatomic localisation of radiopharmaceutical and attenuation correction (AC) of the SPECT images. On HWK-4, the CT scan was acquired in helical mode for faster body acquisitions aimed at reducing the scan time $(\sim 5 \mathrm{~min})$ and thus the radiation exposure $(<2 \mathrm{mSv})$. For brain studies, the CT was acquired in axial mode for improved spatial resolution. As expected, the CT performance was that of a low-grade $\mathrm{CT}\left(3^{\text {rd }}\right.$ generation $)$ with a maximum current of $2.5 \mathrm{~mA}$ and a maximum X-ray tube rotation of 2.6 rpm. In our experience, the CT was not enhanced with oral and/or i.v. contrast agents. As a result, the quality of the $\mathrm{CT}$, as a free-standing device, was considered as suboptimal compared to a fully-diagnostic CT due to respiratory motion, bowel gas artifacts, and reduced contrast-resolution. Despite these inherent limitations, the CT images themselves had a diagnostic value in many clinical circumstances by detecting morphological lesions such as osteolytic/blastic lesions, enlarged lymph nodes, subcutaneous nodules, lung abnormallities, pleural effusion, and abdominal masses. Skeletal lesions tended to be better visualized on CT due to minimal motion during acquisition. For brain studies, a gadolinium-enhanced brain MRI (or contrast-enhanced CT) was found necessary for accurate interpretation. Nonetheless, brain SPECT/CT was used for a fusion with brain MRI on a multimodality imaging Hermes system (www.hermesmedical.com).

\section{Myocardial Perfusion Imaging (MPI)}

Seventy consecutive patients ( 27 female, 43 male, mean age $=62.3$ years \pm 13.5 , mean BMI $\left.=29.8 \mathrm{~kg} / \mathrm{m}^{2} \pm 5.4\right)$ underwent a rest-gated stress ${ }^{99 \mathrm{~m}} \mathrm{Tc}-\mathrm{MIBI}$ SPECT/CT on HWK-4. In 25 (35.7\%) patients, the CT was acquired with 1.0 $\mathrm{mA}$, while the other $45(64.3 \%)$ patients had a $2.5 \mathrm{~mA}$ standard acquisition. OSEM-based iterative reconstruction with attenuation correction (IRAC) significantly increased the normalcy rate from probably normal to definitely normal using a simple and reproducible visual interpretation. In 9 $(13 \%)$ patients, AC with quality control (ACQC) was critical for the appropriate use of IRAC by avoiding misalignmentgenerated artifacts, especially in the anterior wall (Fig. 2). In a subset of 31 consecutive obese patients $\left(B M I \geq 30 \mathrm{~kg} / \mathrm{m}^{2}\right)$, a 3 -reader analysis revealed that IRACQC increased the rates of definite scores (definitely abnormal and definitely normal) from $35.5 \%, 45 \%$, and $55 \%$ to $93.5 \%, 90 \%$, and $58 \%$, for readers R1, R2, and R3, respectively; IRACQC also decreased the rates of unsure scores (probably abnormal, probably normal, and equivocal) from $64.5 \%, 55 \%$, and $45 \%$ to $6.5 \%$, $10 \%$, and $42 \%$ for readers R1, R2, and R3, respectively (Table 2). The mean specificity $(88.3 \%$ vs $71.1 \%)$ and the mean diagnostic accuracy $(86.6 \%$ vs $80.0 \%)$ were consistently improved after IRACQC versus IRNC. Interestingly, a $1.0 \mathrm{~mA}$ acquisition was found feasible for an effective AC without compromising the diagnostic accuracy for detection of CAD (Graph 1). Fig. (2) illustrates the clinical impact of AC quality control for correction of artifact-generated misalignment.

\section{Differentiated Thyroid Cancers (DTC)}

Among the 42 patients ( 9 male, 33 female, mean age $=50$ years \pm 15.9 ) with DTC (29 papillary carcinomas, 2 follicular carcinomas, 8 papillary carcinomas with follicular variant, and 3 Hürtle cell carcinomas), SPECT/CT allowed confident localisation and characterisation of equivocal foci seen on planar whole-body in $36(85.7 \%)$ cases. These ${ }^{131} \mathrm{I}$-avid foci included physiological uptake patterns in $22(52.4 \%)$ patients corresponding to the thyroid bed, the thyroglossal tract, the salivary glands, the thymus, the esophagus, the rectum, the

Table 2. SPECT-CT MPI in Obese Patients - Diagnostic Performances

\begin{tabular}{|c|c|c|c|c|}
\hline & IRNC vs IRAC & $\%$ Sensitivity & $\%$ Specificity & \% Diagnostic Accuracy \\
\hline $\mathbf{R 1}$ & $\begin{array}{c}\text { A- } 1 ; 2 ; 3 ; 4 ; 5 \\
\text { B- } 1 ; 2 ; 3 ; 4 ; 5 \\
\text { C- } 1 ; 2 ; 4 ; 5\end{array}$ & $\begin{array}{c}93 \% \text { vs } 78 \% \\
93 \% \text { vs } 100 \% \\
100 \% \text { vs } 100 \%\end{array}$ & $\begin{array}{l}100 \% \text { vs } 100 \% \\
100 \% \text { vs } 100 \% \\
100 \% \text { vs } 100 \%\end{array}$ & $\begin{array}{c}96 \% \text { vs } 90 \% \\
96 \% \text { vs } 100 \% \\
100 \% \text { vs } 100 \%\end{array}$ \\
\hline $\mathbf{R 2}$ & $\begin{array}{c}\text { A- } 1 ; 2 ; 3 ; 4 ; 5 \\
\text { B- } 1 ; 2 ; 3 ; 4 ; 5 \\
\text { C- } 1 ; 2 ; 4 ; 5\end{array}$ & $\begin{array}{l}92 \% \text { vs } 64 \% \\
90 \% \text { vs } 63 \% \\
90 \% \text { vs } 63 \%\end{array}$ & $\begin{array}{l}72 \% \text { vs } 94 \% \\
62 \% \text { vs } 85 \% \\
72 \% \text { vs } 94 \%\end{array}$ & $\begin{array}{l}80 \% \text { vs } 80 \% \\
71 \% \text { vs } 77 \% \\
78 \% \text { vs } 82 \%\end{array}$ \\
\hline $\mathbf{R 3}$ & $\begin{array}{c}\text { A- } 1 ; 2 ; 3 ; 4 ; 5 \\
\text { B- } 1 ; 2 ; 3 ; 4 ; 5 \\
\text { C- } 1 ; 2 ; 4 ; 5\end{array}$ & $\begin{array}{c}100 \% \text { vs } 93 \% \\
100 \% \text { vs } 100 \% \\
100 \% \text { vs } 100 \%\end{array}$ & $\begin{array}{l}47 \% \text { vs } 76 \% \\
40 \% \text { vs } 70 \% \\
47 \% \text { vs } 76 \%\end{array}$ & $\begin{array}{l}71 \% \text { vs } 84 \% \\
61 \% \text { vs } 80 \% \\
67 \% \text { vs } 86 \%\end{array}$ \\
\hline
\end{tabular}

Abbreviations: A; B; C: 3 scenario for calculation of diagnostic performances based on a 5-point scale analysis with normal MPIs in green and abnormal MPIs in red (A; B) or 4-point scale analysis without equivocal scores (C). R1, R2, R3: readers 1, 2, and 3 . 


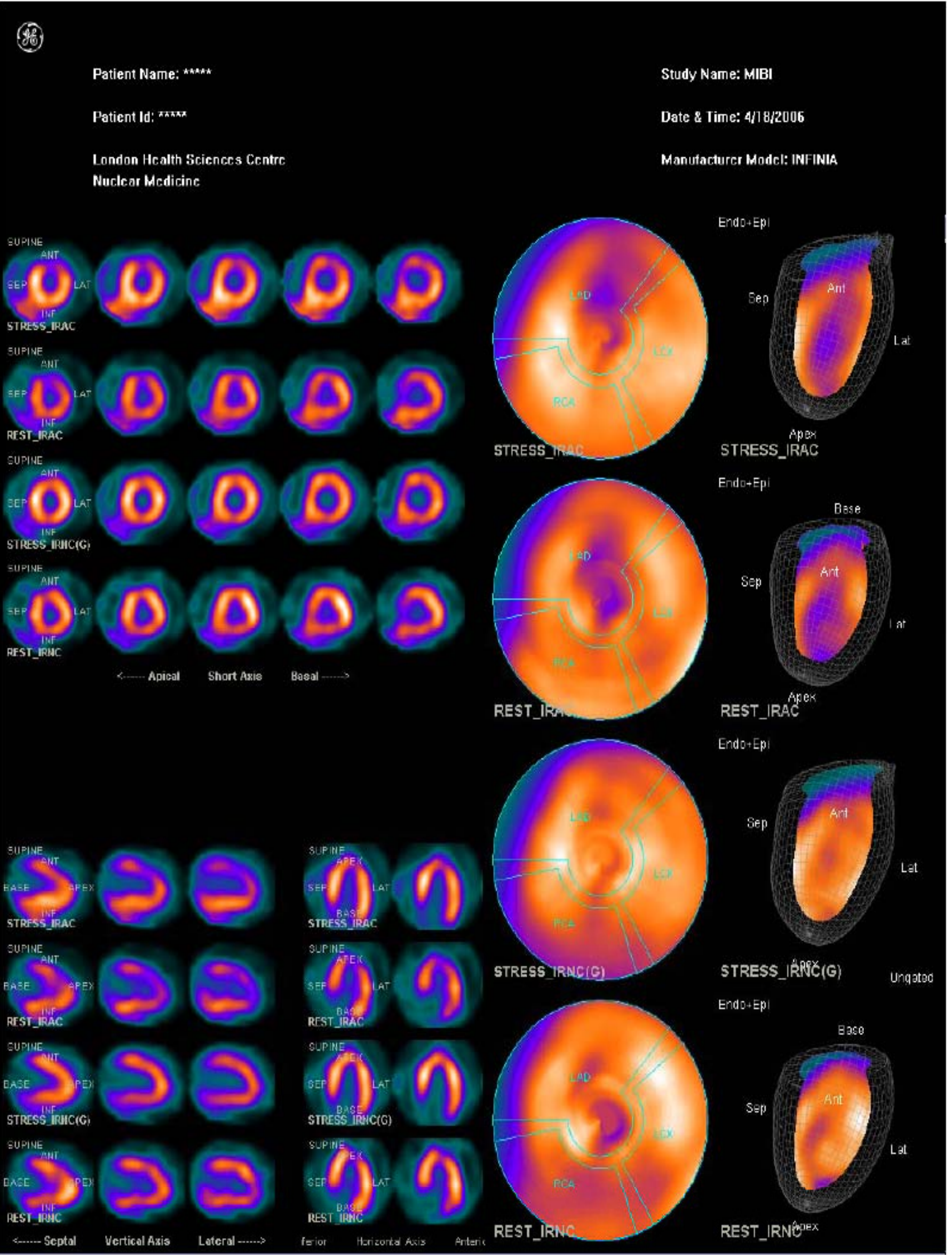


(Fig. 2) contd...

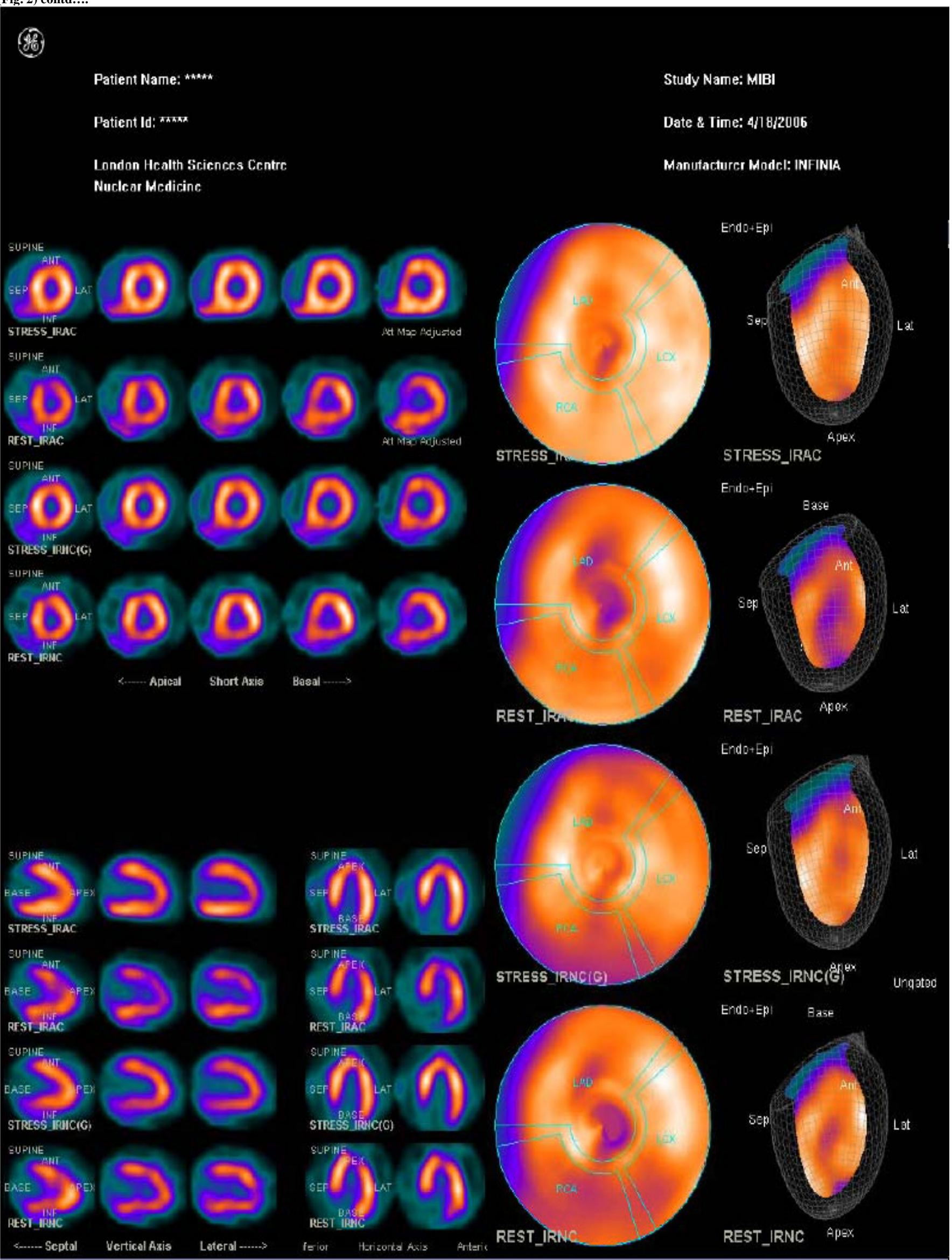




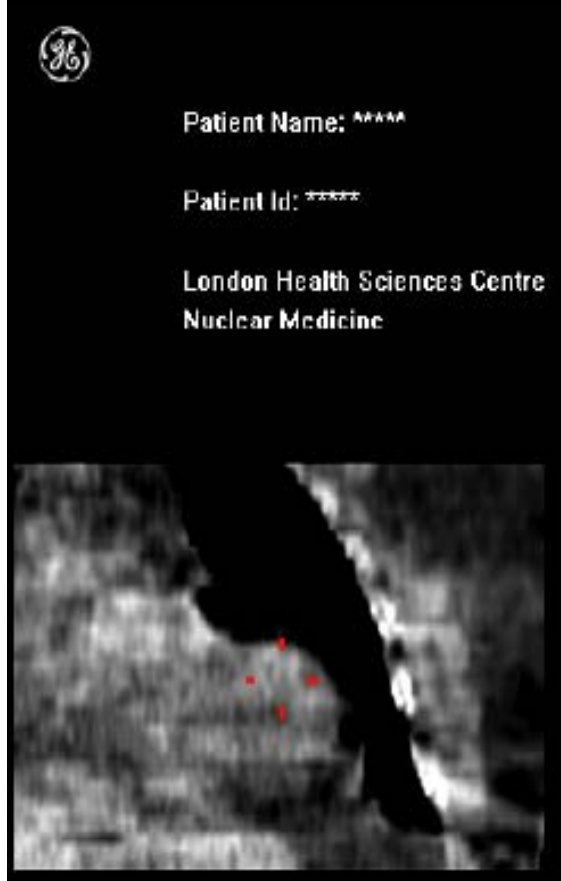

cT Coronals

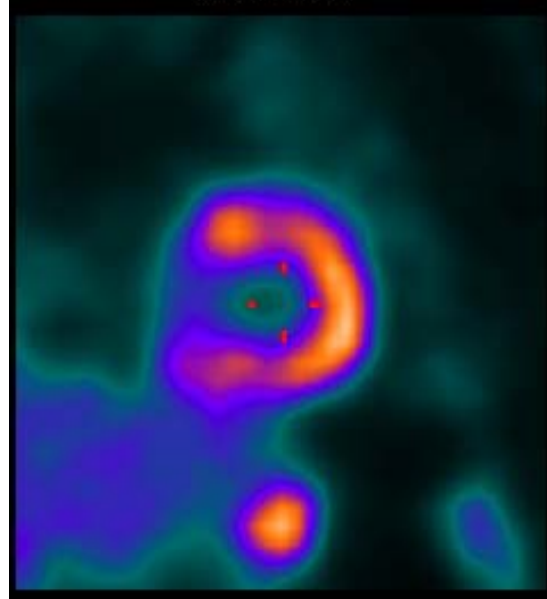

NM Coronals

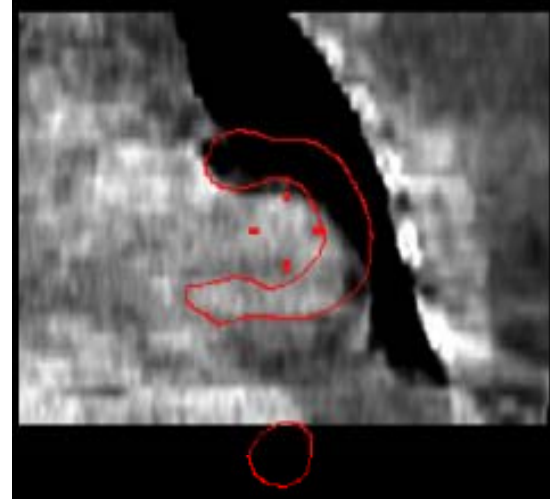

Fused Coronals

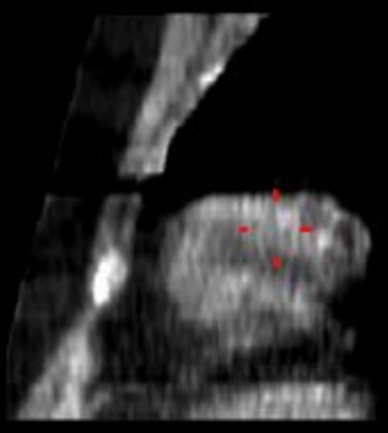

CT sagittals

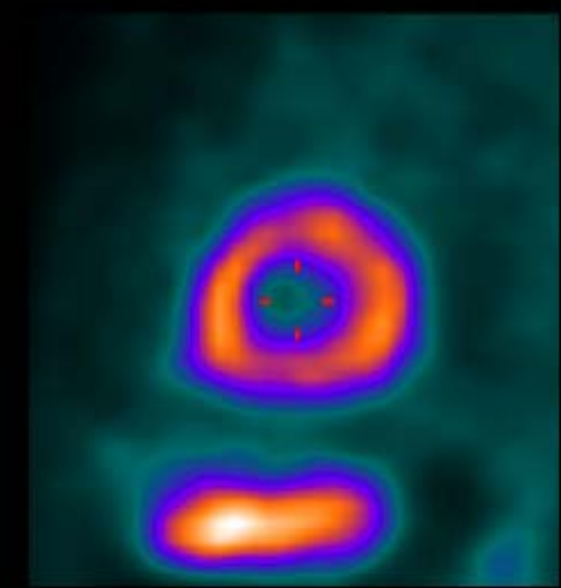

NM Sagittals

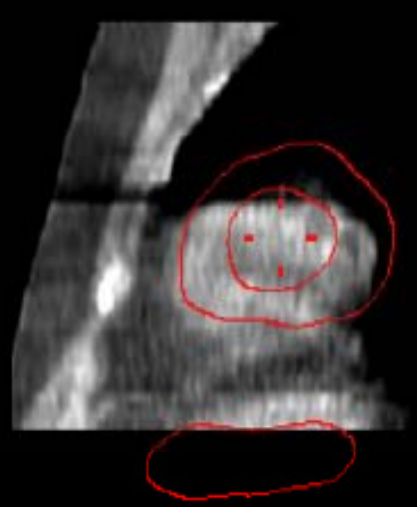

Fused Saglttals

1
Study Name: MIBI

Date \& Time: $4 / 18 / 2006$

Manufacturer Model: INIFINIA



CT Transaxials

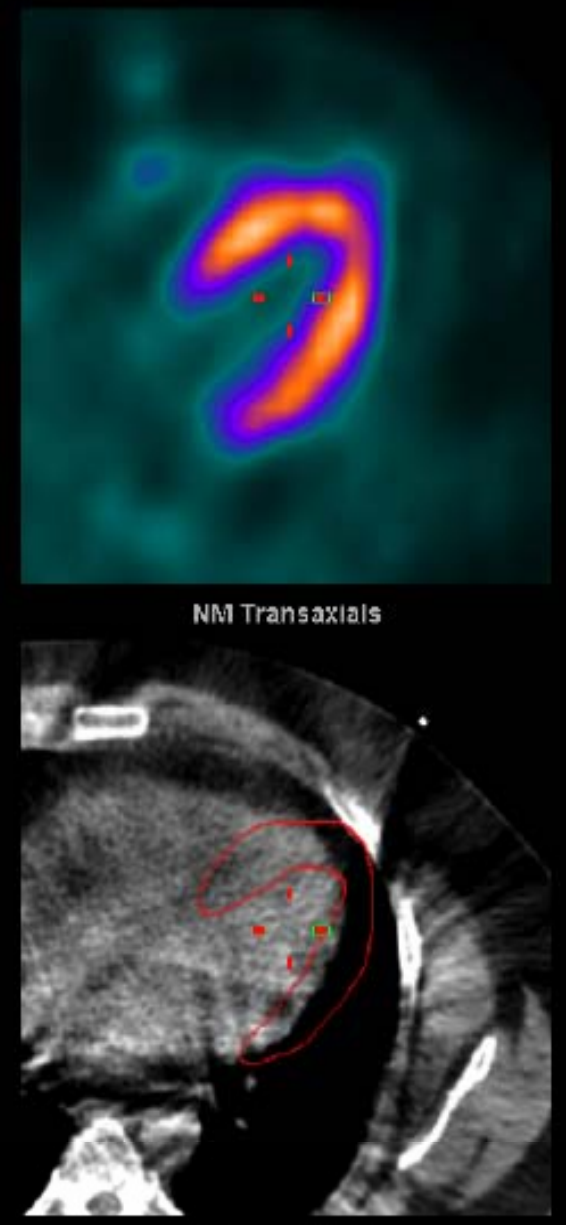

Fused Transaxlals 
(Fig. 2) contd...

(39)

Patient Name: thank

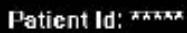

London Health Siciences Centre Nuclear Medicine

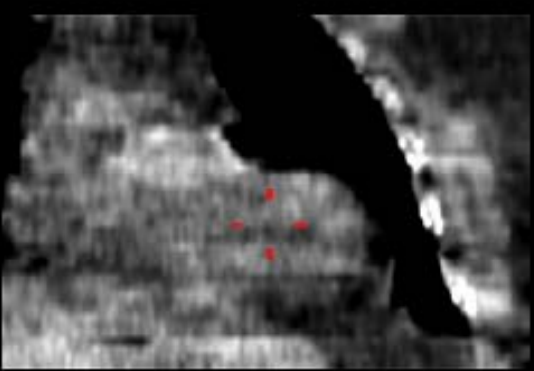

CT Coronals

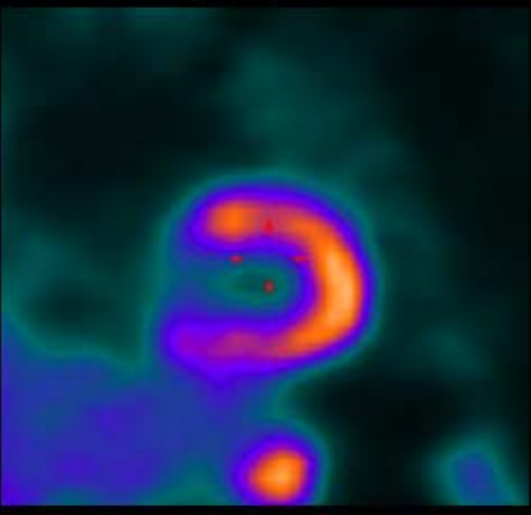

NM Coronals

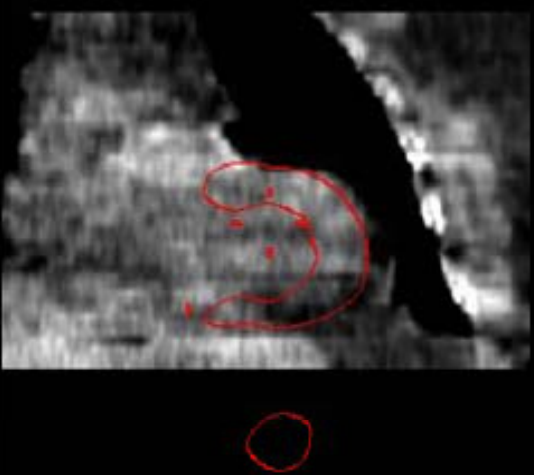

Fused Coronals

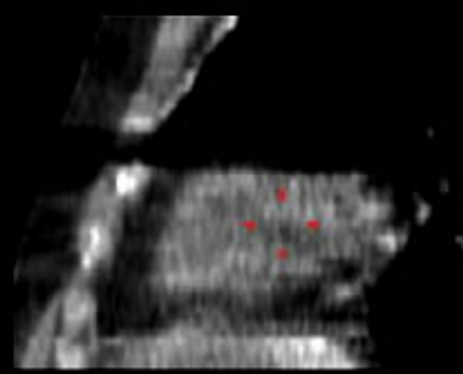

CT Sagittals

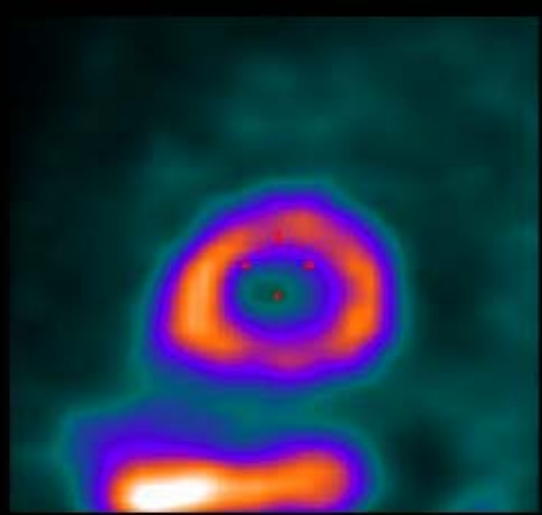

NM Sagittals
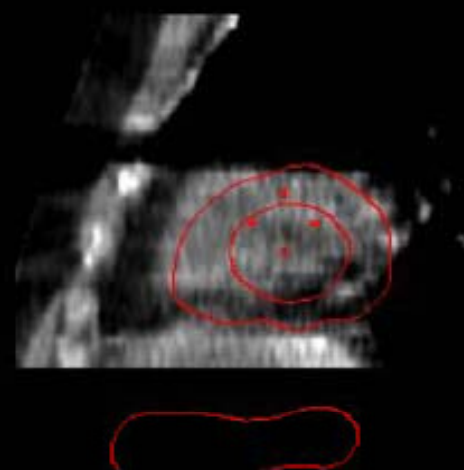

Fused Sagittals
Study Name: MIB

Date \& Time: 4/78/2006

Manufacturcr Model: INFINIA

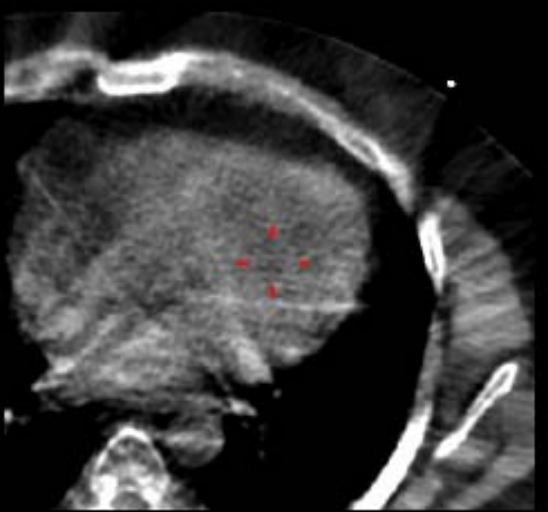

CT Transaxials

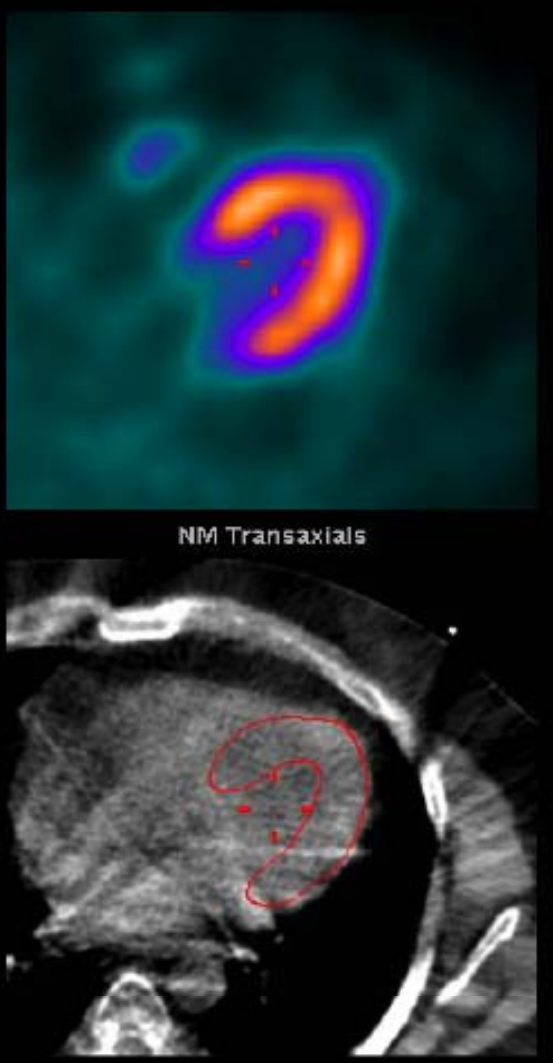

Fused Transaxials

Fig. (2). A 44 year-old male with a low pretest likelihood of CAD was referred for atypical chest pains. BMI $=38.09 \mathrm{~kg} / \mathrm{m}^{2} . \mathrm{A}$ rest $/ \mathrm{gated}-$ stress MPI study was performed in a 1-day ${ }^{99 \mathrm{~m}} \mathrm{Tc}-\mathrm{MIBI}$ protocol following a Dipyridamole with bicycle stress test. On IRNC images, a relatively homogenous tracer uptake was noted except for the inferior wall, which was suggestive of infra-diaphragmatic attenuation. After IRAC but no ACQC, complete correction for the inferior wall attenuation was seen, while an artifactual defect was generated on the anteroapical wall (left top). On stress images, ACQC revealed a significant shift (left bottom) between the CT and SPECT data (> 1.5 pixels), which led to manual realignment (right bottom). After IRAC and ACQC, complete correction for the inferior wall attenuation was observed without AC-generated artifacts (right top). LVEF was normal (65\%) with no TID (1.13). This MPI study was interpreted as a definitely normal study based on IRAC-ACQC vs IRNC data. 


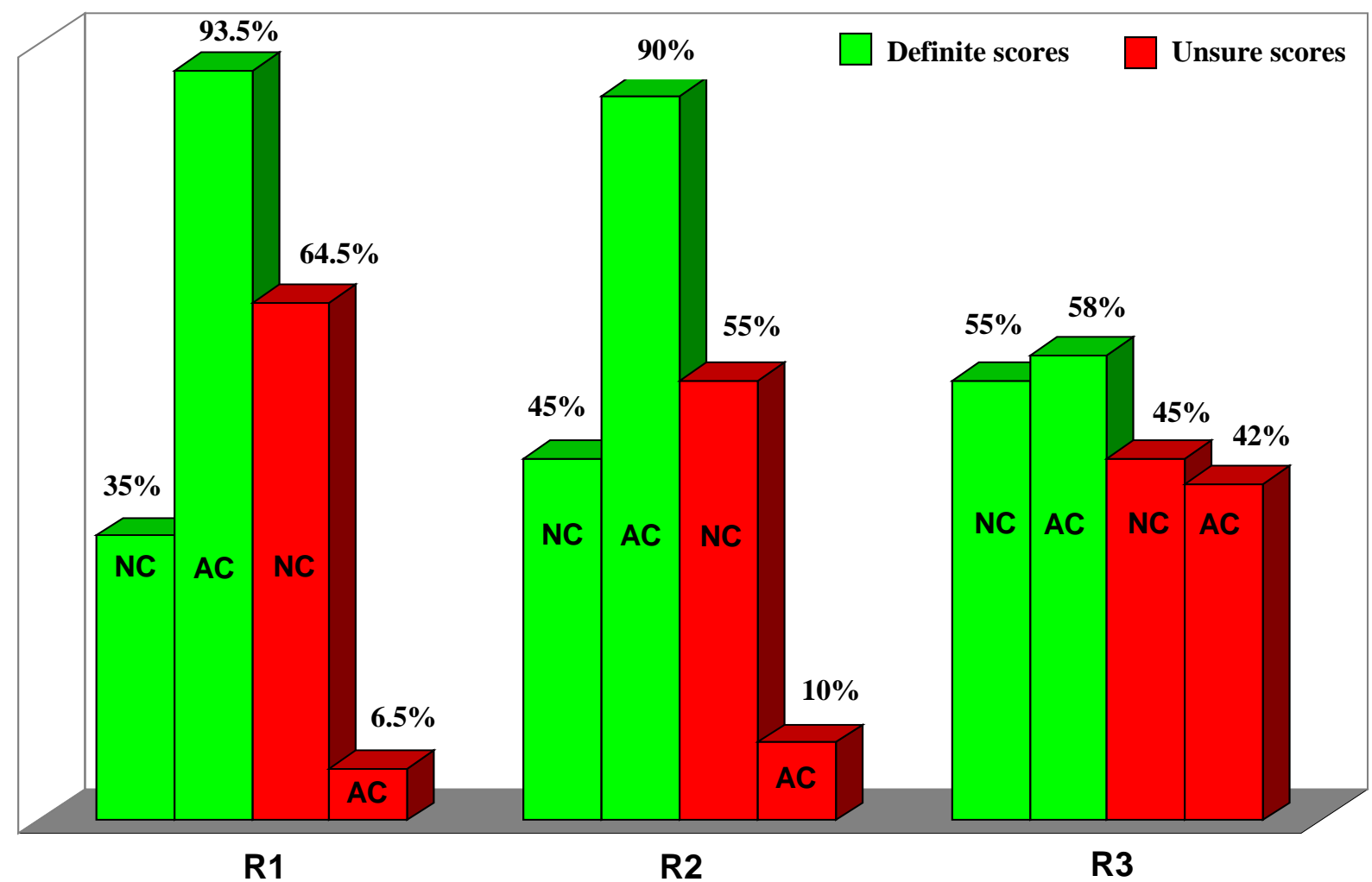

Abbreviations: NC: non-attenuation corrected images; AC: attenuation corrected images; Definite scores: definitely normal + definitely abnormal; Unsure scores: probably normal + probably abnormal + equivocal

Graph 1. SPECT-CT MPI in obese patients - interpretation confidence.

uterus, and benign uptake patterns in 14 (33.3\%) patients related to dental work, gastro-esophageal reflux disease, diaphragmatic hernia, and mastoiditis. Pathological uptake patterns were also better identified on SPECT/CT than planar WBS including ${ }^{131}$ I-avid brain, lung, nodal, or skeletal metastases. In 6 (14.3\%) patients, SPECT/CT helped to guide complementary imagingwork-up such as high-resolution neck ultrasound, diagnostic chest CT, and brain MRI (Table 3).

\section{Neuroendocrine Tumours (NET)}

Seventy patients with various types of neuroendocrine tumors (carcinoids, glucagonoma, medullary thyroid carcinoma, gastrinoma, MEN I syndrome, neuroblastoma, phechromocytoma, adrenal cortical carcinoma, pancreatic carcinoma, Goblet cell carcinoma) underwent ${ }^{131}$ I-MIBG $(\mathrm{n}=13),{ }^{123} \mathrm{I}-\mathrm{MIBG}(\mathrm{n}=6)$, and ${ }^{111}$ In-Octreotide $(\mathrm{n}=51)$ planar WBS and SPECT/CT scans for pre-treatment staging, restaging, and post-therapy evaluation. In these clinical indications, SPECT/low-dose multislice CT was found particularly useful for accurate anatomic localisation, differentiation between physiological and pathological uptakes, and detection of abdominal foci not seen on planar whole-body scan including nodal, liver, peritoneal, and splenic lesions. The most significant contribution of CT was the detection of unknown lung lesions (small foci or non tracer avid metastases). However, the quality of the CT scan was found suboptimal for accurate characterisation of liver metastases due to respiratory motion artifacts and absence of contrast enhancement. Even if infra- and supradiaphragmatic disease was found on planar WBS, the SPECT/CT scan duration ( $\pm 37 \mathrm{~min} / \mathrm{FOV})$, however, prevented the acquisition of more than one SPECT/CT

Table 3. Impact of SPECT/CT on Management of Differentiated Thyroid Cancers

\begin{tabular}{|c|c|c|}
\hline $\begin{array}{c}\text { Physiological or Benign Uptakes vs Pathological } \\
\text { Uptakes with No Further Work-Up }\end{array}$ & $\begin{array}{l}\text { Accurate Anatomic Localisation of } \\
{ }_{131} \text { I-avid Metastases }\end{array}$ & Appropriate Guidance of Further Work-Up \\
\hline $\begin{array}{l}\text { - Head : hair contamination, mastoiditis, dental works } \\
\text { - Neck : thyroid bed, thyroglossal tract, salivary glands } \\
\text { - Mediastinum : esophagus, thymus, gastro-esophageal } \\
\text { reflux } \\
\text { - Lung : diaphragmatic hernia } \\
\text { - Abdomen : colon } \\
\text { - Pelvis : rectum, uterus }\end{array}$ & $\begin{array}{l}\text { - Brain } \\
\text { - Lung } \\
\text { - Bone } \\
\text { - Lymph nodes (cervical and supra- } \\
\text { clavicular lymph nodes) }\end{array}$ & $\begin{array}{l}\text { - Cervical lymph nodes : high-resolution } \\
\text { ultrasound } \\
\text { - Lung abnormalities : diagnostic CT } \\
\text { - Brain lesions : MRI }\end{array}$ \\
\hline
\end{tabular}


study. Hence, most NET patients had only an abdominal or rarely a chest SPECT/CT in daily routine (Table 4).

\section{Bone Imaging}

In the 51 patients ( 34 oncology patients, and 17 nononcology patients) who had a conventional whole-body bone scan with ${ }^{99 \mathrm{~m}} \mathrm{Tc}-\mathrm{MDP}$, SPECT/CT was found to be of clinical value for clarifying equivocal foci. HWK -4 led to a confident interpretation for a faster or final diagnosis in $82 \%$ of oncology patients, thereby, avoiding the need of further investigations. This was particularly true in the spine where the low-dose multislice CT differentiated disc and facet joints degenerative disease from osteolytic/blastic metastases. Oncology patients benefited diagnostically from Bone Evolution; a half-time acquisition with resolution recovery, which permitted scanning of the entire spine over 2 FOVs ( 76 $\mathrm{cm}$ ) in less than $30 \mathrm{~min}$ while improving the quality of the SPECT scan (Fig. 3). Nonetheless, the contrast-resolution of HWK -4 was found suboptimal for detection of bone marrow involvement, thereby, requiring a further MRI. SPECT/CT was found of added-value in 34\% of non-oncology patients referred for lower back pain, neck pain, chest pain, joints pain, or suspicion of osteomyelitis; low-dose multislice CT allowed more confident diagnosis of spine and joint benign diseases including degenerative disease (facet joints osteoarthritis, space narrowing, osteophyte formation, Schmorl's node patterns), avascular necrosis of the hip, entesopathy, enchondroma, scoliosis, disk hernia, and rib fracture callus. Importantly, a close collaboration with a musculo-skeletal radiologist and CT training for the nuclear medicine physicians were found required for the optimal use of SPECT/CT in benign bone diseases (Table $\mathbf{5}$ ).

\section{Sentinel Lymph Node Detection (SLN)}

In the 12 patients $(9$ female, 3 male, mean age $=57.67$ years $\left.\pm 14.6, \mathrm{BMI}=27.2 \mathrm{Kg} / \mathrm{m}^{2} \pm 5.3\right)$ with a breast cancer $(n=7)$, a malignant melanoma $(n=4)$, and a penile cancer $(\mathrm{n}=1)$ imaged for SLN detection in a 2-day protocol with ${ }^{99 \mathrm{~m}} \mathrm{Tc}$-rhenium cystein colloids (mean activity $=52.5 \mathrm{MBq}$ ), SPECT/low-dose multislice CT significantly improved the accurate 3D localisation of hot nodes compared to $2 \mathrm{D}$ planar views. In breast cancer, SLNs could be precisely localised at the levels I, II, and III of the axilla, and in extra-axillary regions such as the intercostal and internal mammary lymphatic chains. In malignant melanoma and penile cancer, SPECT/CT also helped detect deep SLNs located in the iliofemoral and mesenteric lymphatic chains, and allowed to ruling out a doubtful focus on planar views. CT-based anatomic localisation and attenuation correction allowed the detection of additional relevant nodes not seen on planar imaging alone, particularly in 2 out of 4 overweight patients $(25<\mathrm{BMI} \leq 29.9)$ and 3 out of 4 obese patients (BMI $\geq 30)$. Tables 6 and 7 detail the total numbers, the anatomic sites, and the pathological status of SLNs detected on SPECT/CT versus planar imaging.

\section{ProstaScint ${ }^{\circledR}$ Imaging}

In the 27 consecutive prostate cancer patients referred for a suspicion of recurrence based on rising PSA post-therapy $(n=22)$, or for a salvage work-up in high-risk patients with PSA $<1.0 \quad \mathrm{ng} / \mathrm{ml} \quad(\mathrm{n}=4), \quad$ or for initial staging $(\mathrm{n}=1)$, SPECT/CT ProstaScint ${ }^{\circledR}\left({ }^{111}\right.$ In-Capromab Pendetide) studies were found particularly useful for detection of local

Table 4. Added-Value of Infinia-Hawkeye -4 in ${ }^{67} \mathrm{Ga},{ }^{123 / 131}$ I-MIBG, and ${ }^{111}$ In-Octreotide Studies

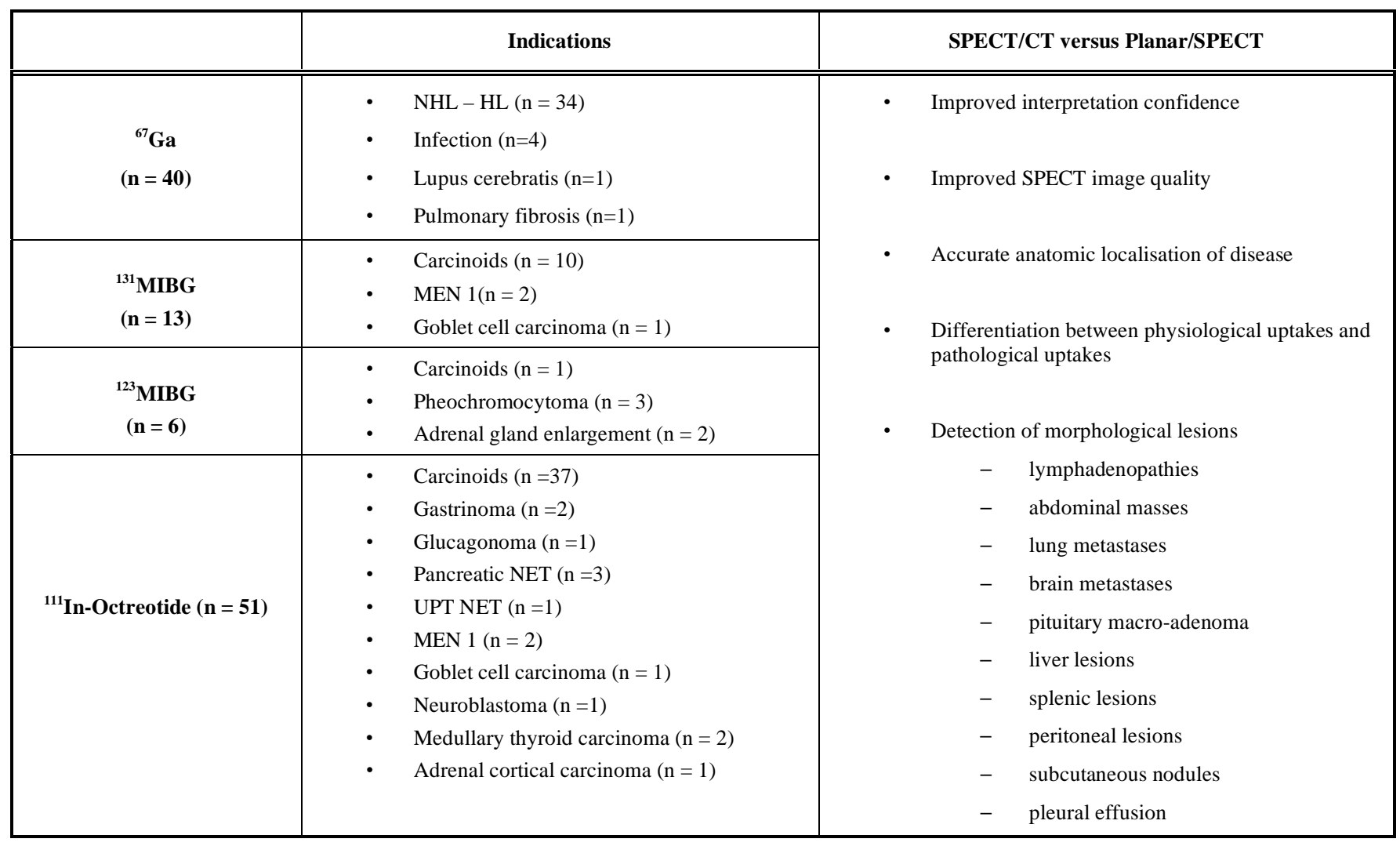


Table 5. Added-Value of SPECT/CT in Equivocal Planar Whole-Body Bone Scan

\begin{tabular}{|c|c|c|}
\hline & Indications & Clinical Implications \\
\hline Oncology Patients $(n=34)$ & $\begin{array}{ll}\text { - } & \text { Breast Cancer }(\mathrm{n}=13) \\
\text { - } & \text { Lung Cancer }(\mathrm{n}=10) \\
\text { - } & \text { Prostate Cancer }(\mathrm{n}=7) \\
\text { - } & \text { Renal Cancer }(\mathrm{n}=1) \\
\text { - } & \text { Lymphoma }(\mathrm{n}=1) \\
\text { - } & \text { Cervical Cancer }(\mathrm{n}=1) \\
\text { - } & \text { Giant cell tumour }(\mathrm{n}=1)\end{array}$ & $\begin{array}{l}\text { - Improved interpretation confidence } \\
\text { - } \quad \text { Improved specificity } \\
\text { - } \quad \text { Better anatomic localisation } \\
\text { - } \quad \text { Detection of morphological bone lesions } \\
-\quad \text { benign : spine DGD, enchondroma, fractures } \\
\text { - } \quad \text { Metastases : osteolytic/blastic metastases } \\
\text { Final or faster diagnosis after SPECT/CT }=82 \%\end{array}$ \\
\hline $\begin{array}{l}\text { Non-Oncology Patients } \\
\qquad(\mathbf{n}=\mathbf{1 7})\end{array}$ & $\begin{array}{ll}\text { - } & \text { Lower Back Pain }(n=8) \\
\text { - } & \text { Neck Pain }(n=1) \\
\text { - } & \text { Chest Pain }(n=1) \\
\text { - } & \text { Joint Pain }(n=6) \\
\text { - } & \text { Osteomyelitis }(n=1)\end{array}$ & 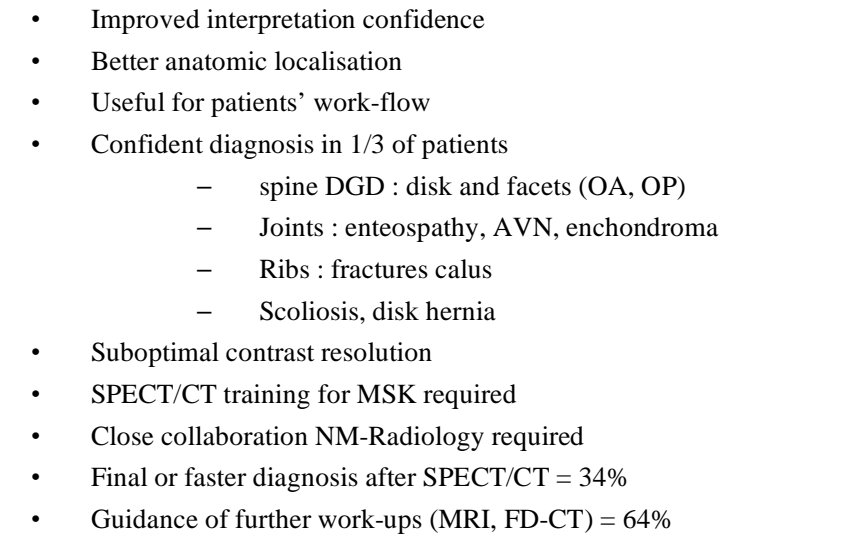 \\
\hline
\end{tabular}

pelvic disease (i.e. prostate bed, seminal vesicles, lymph nodes, bladder wall) missed on planar WBS or equivocal on SPECT alone. Combined imaging also helped confirm extrapelvic disease involving para-aortic or cervical lymph nodes. CT anatomy and attenuation correction significantly improved the interpretation confidence compared to planar/SPECT imaging alone (Table 8); SPECT/CT allowed anatomic localisation of physiological uptakes (i.e. vessels, gut, bone marrow) versus pathological uptakes (i.e. local, regional, and/or distant recurrences). In addition, ProstaScint ${ }^{\circledR}$ SPECT/CT image quality with CT-based attenuation correction allowing increase of signal-tobackground ratio was significantly improved over that of planar/SPECT ProstaScint ${ }^{\circledR} / \mathrm{RBC}$ dual-isotope imaging modality. In comparison to planar/SPECT imaging, SPECT/CT significantly impacted the treatment choices in $21(78 \%)$ patients in terms of salvage or adjuvant external radiation therapy versus hormone systemic therapy and/or surveillance, especially in patients with slow rise of PSA $(<$ $4 \mathrm{ng} / \mathrm{ml}$ ). Fig. (4) illustrates the impact of SPECT/CT in ProstaScint ${ }^{\circledR}$ imaging for detection of small pelvic recurrence and treatment planning.

\section{${ }^{67}$ Ga Imaging}

In the 40 patients evaluated with ${ }^{67} \mathrm{Ga}$ scan (34 Hodgkin and non-Hodgkin lymphomas, 4 suspicions of infection, 1 lupus cerebratis, 1 pulmonary fibrosis), SPECT/CT allowed a faster or final more confident diagnosis in $19(47.5 \%)$ cases. The low-dose multislice CT scan revealed morphological lesions in 19 patients $(47.5 \%)$ and suggested a morphological diagnosis in another 14 (35.0)\%. In comparison with planar WBS and SPECT alone, SPECT/CT was of incremental value in 23 patients $(57.5 \%)$, particularly for accurate anatomic localisation of disease, and differentiation between physiological and pathological uptakes. Of note, the total scan duration for a planar WBS plus total body SPECT/CT was long, about 150 minutes per patient (Table 4).

\section{RBC Imaging}

In the 11 patients with liver or splenic hemangiomas and splenosis, SPECT/CT was found particularly useful for accurate localisation of hot spots related to morphological lesions versus vascular uptakes (aorta, intra-hepatic vessels). Similarly, SPECT/CT allowed precise detection of functional splenic remnants, which were either missed by diagnostic CT or MRI or ill-defined by SPECT alone. Lowdose CT was found suboptimal for detection of small hepatic lesions $(<1.5 \mathrm{~cm})$. Table 9 summarizes the added-value of the Infinia-Hawkeye -4 in $99 \mathrm{mTc}$-red blood cell imaging.

\section{WBC Imaging}

In comparison to planar views and SPECT alone, SPECT/CT provided incremental value for accurate localisation of infection within bone structures, thereby, allowing differentiation between osteomyelitis and soft tissue infection in 15 subjects. Twenty four ${ }^{99 \mathrm{~m}} \mathrm{Tc}-\mathrm{HMPAO}-$ WBC scans were performed in 23 consecutive patients with Crohn's disease (CD). SPECT/CT improved the interpretation confidence by increasing the definite scores from 10 to 20 definitely positive/negative scans, and by reducing the unsure scores from 14 to 4 probably positive/negative and equivocal scans, when compared to 


\section{(19:6)}

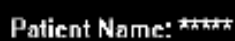

Patient Id: ${ }^{\text {*ank }}$

London Health Sciences Centre

Nuclear Medicine

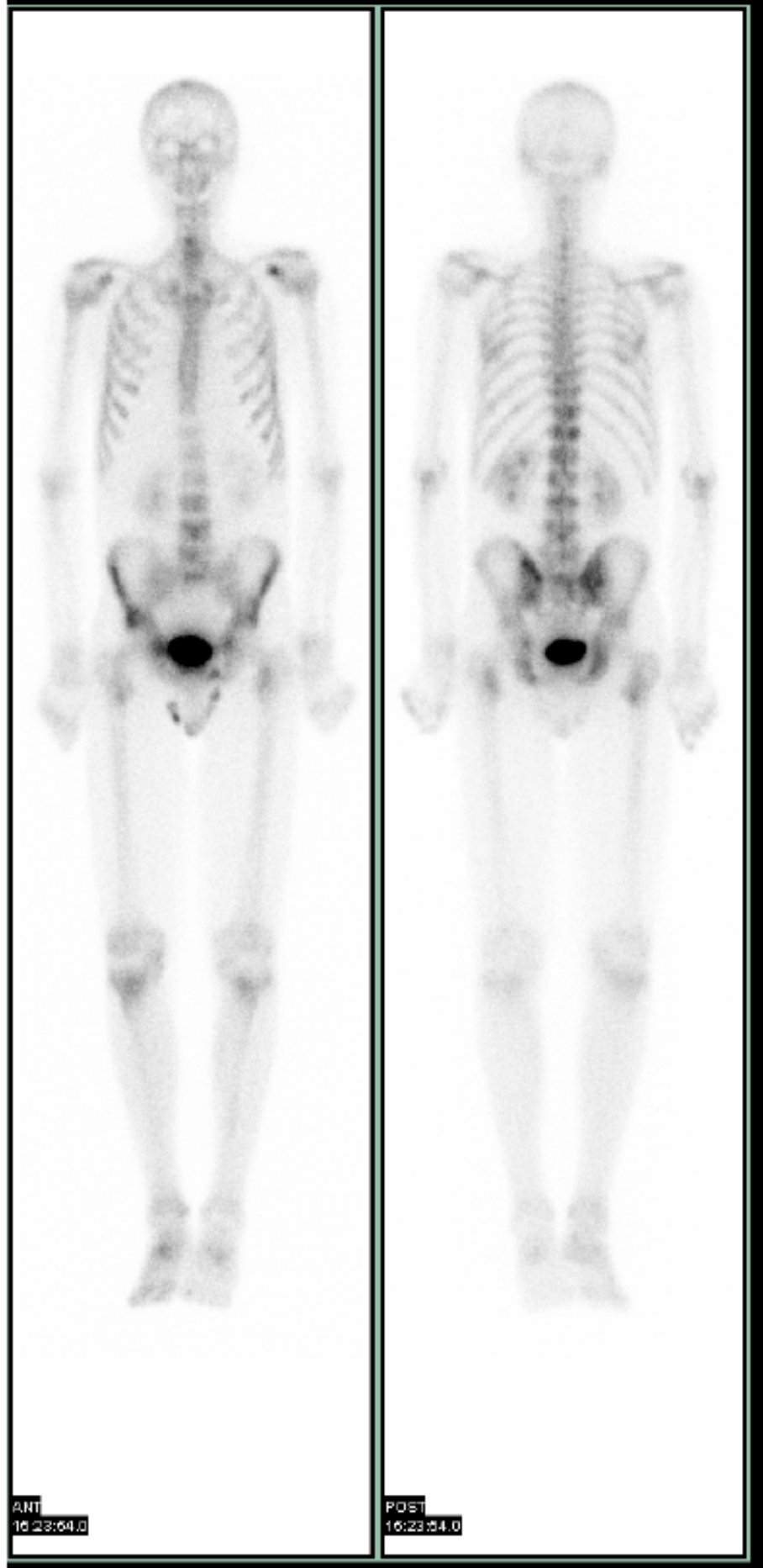

Study Name: NM bone scan

Date \& Time: 5/2/2006

Manulacturer Model: INFINIA 


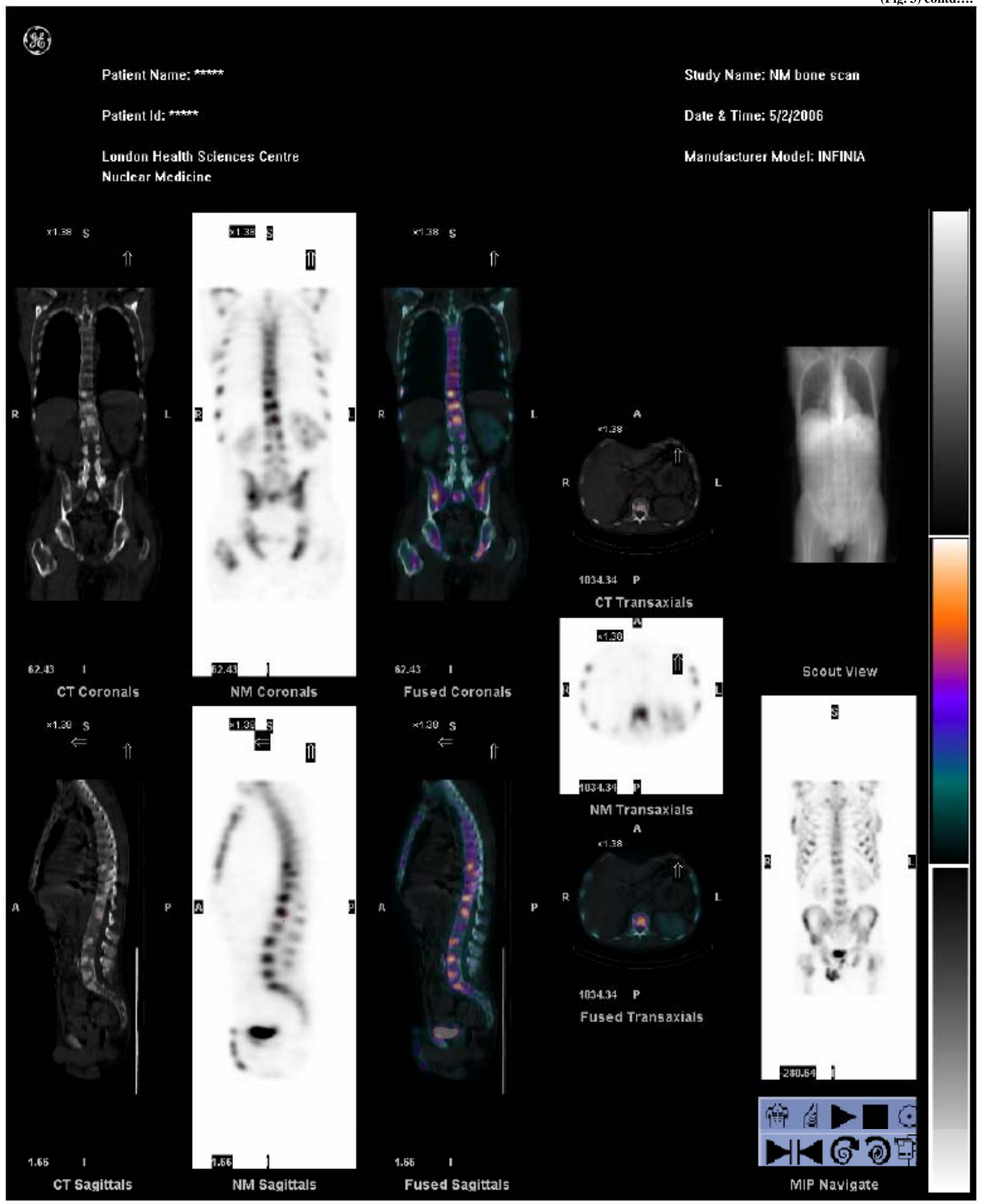


(Fig. 3) contd...

(9:

Patient Name:

Patient Id: "*ak*

London Health Sciences Centre Nuclear Medicine

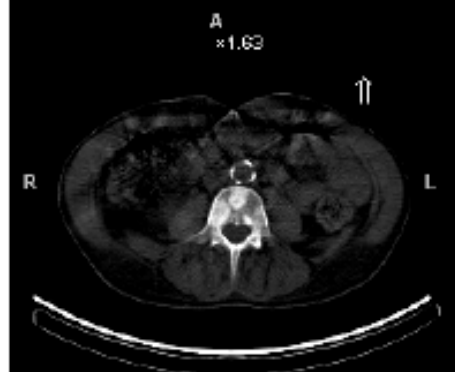

927.28

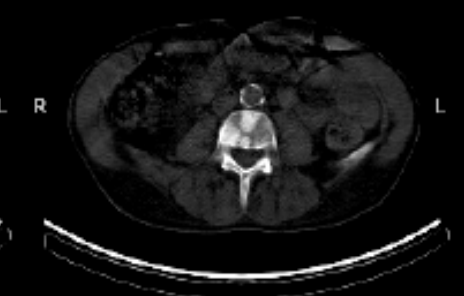

92286 P
A

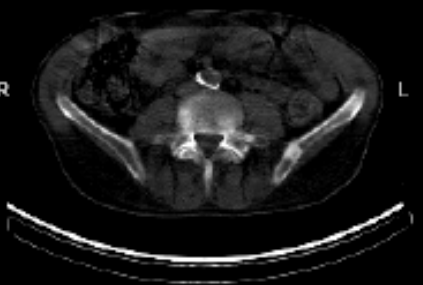

$\begin{array}{ll}905.19 & \text { P } \\ & \text { A }\end{array}$
Study Name: NM bone scan

Date \& Time: 5/2/2006

Manufacturer Model: INFINIA

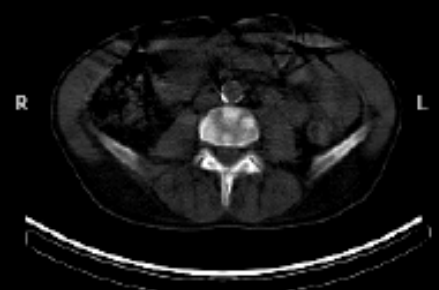

918.44 P

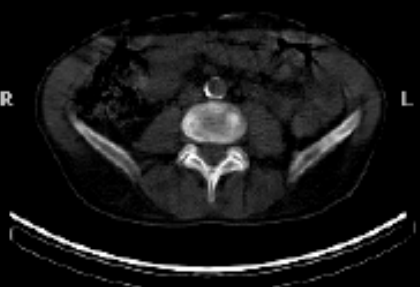

914.102 P

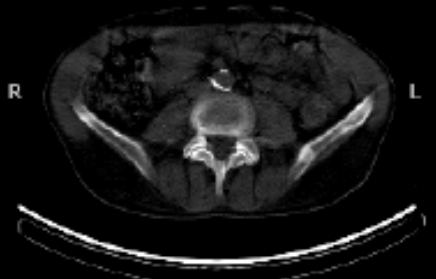

909.60

P

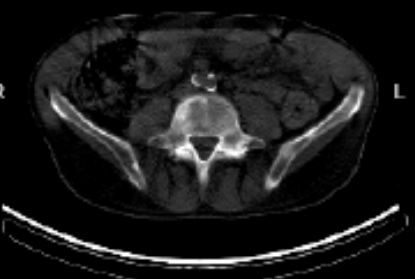

900.77

P

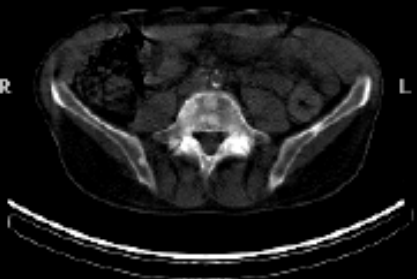

896.35
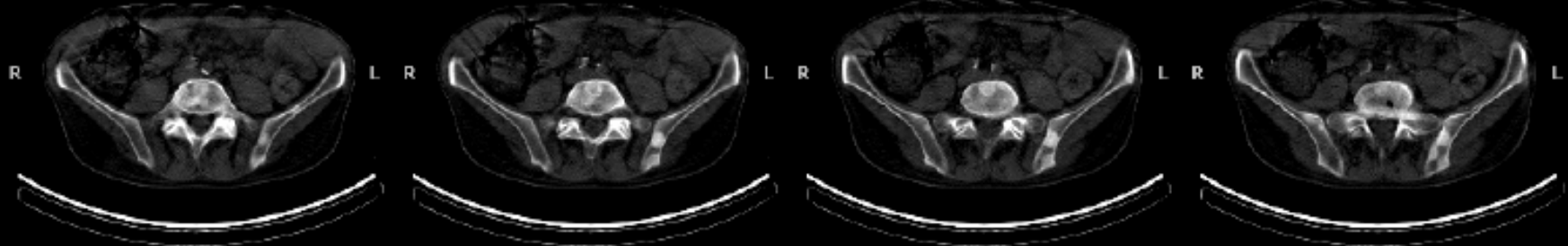

691.93

6อง.ร1 P

683. 09

678.67 P

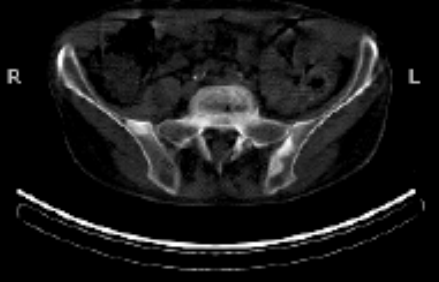

874.25

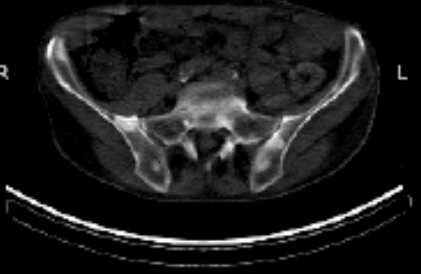

969.85

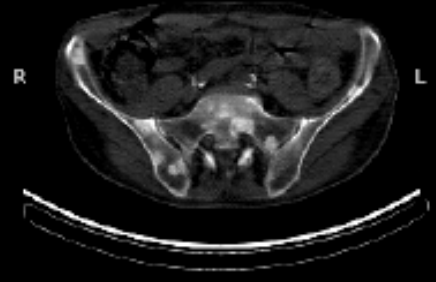

065.41

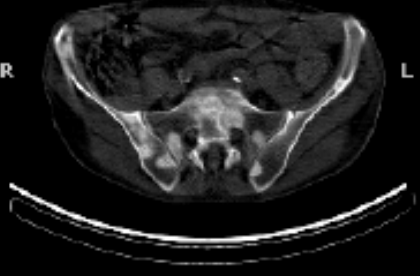

860.99 $P$

CT Transaxials

Fig. (3). A 47-year old patient with a small-cell lung cancer had a bone scan for pre-treatment staging purposes. Planar whole-body scan showed a suspicious heterogeneous uptake involving the dorsal spine and the lumbar spine as well as the sacro-iliac joints. Bone Evolution SPECT/CT acquisition and reconstruction over the entire spine (2 FOVs in $28 \mathrm{~min}$ ) confirmed the presence of vertebral and sacro-iliac sclerotic bone metastases (top). The low-dose multislice CT also detected small iliac and spine lesions not seen on SPECT (bottom). 
Table 6. SPECT/CT Versus Planar Imaging for Sentinel Lymph Node Detection

\begin{tabular}{|c|c|c|c|c|c|c|c|}
\hline Patients & Primary Tumour & $\begin{array}{c}\text { Age } \\
\text { (Years) }\end{array}$ & $\underset{\left(\mathrm{Kg} / \mathrm{m}^{2}\right)}{\mathrm{BMI}}$ & $\begin{array}{l}\text { Number of SLNs on } \\
\text { Planar Imaging }\end{array}$ & $\begin{array}{l}\text { Number of SLNs } \\
\text { on SPECT/CT }\end{array}$ & $\begin{array}{c}\text { Number of SLNs at } \\
\text { Surgery and Pathology }\end{array}$ & $\begin{array}{c}\text { SLN Status (CT } \\
\text { Greatest Diameter) }\end{array}$ \\
\hline 1 & Penile Cancer & 56 & 35.5 & 2 & 3 & NA & $\mathrm{SLN}-(<10 \mathrm{~mm})$ \\
\hline 2 & Malignant Melanoma & 66 & 32.8 & 1 & 2 & 1 & $\mathrm{SLN}-(<10 \mathrm{~mm})$ \\
\hline 3 & Breast Cancer & 52 & 30.6 & 1 & 3 & 5 & $\mathrm{SLN}+(14.7 \mathrm{~mm})$ \\
\hline 4 & Malignant Melanoma & 42 & 34.3 & 2 & 2 & 4 & $\mathrm{SLN}-(<10 \mathrm{~mm})$ \\
\hline 5 & Malignant Melanoma & 43 & 18.8 & 4 & 4 & 2 & $\mathrm{SLN}-(<10 \mathrm{~mm})$ \\
\hline 6 & Malignant Melanoma & 84 & 27.0 & 5 & 5 & 4 & $\mathrm{SLN}+(<10 \mathrm{~mm})$ \\
\hline 7 & Breast Cancer & 51 & 25.2 & 2 & 2 & 2 & $\mathrm{SLN}-(<10 \mathrm{~mm})$ \\
\hline 8 & Breast Cancer & 67 & 19.7 & 2 & 2 & 3 & $\mathrm{SLN}-(<10 \mathrm{~mm})$ \\
\hline 9 & Breast Cancer & 77 & 23.1 & 1 & 1 & 1 & $\mathrm{SLN}-(<10 \mathrm{~mm})$ \\
\hline 10 & Breast Cancer & 69 & 29 & 1 & 4 & 3 & $\mathrm{SLN}-(<10 \mathrm{~mm})$ \\
\hline 11 & Breast Cancer & 42 & 25.5 & 1 & 2 & 2 & $\mathrm{SLN}-(<10 \mathrm{~mm})$ \\
\hline \multirow[t]{2}{*}{12} & Breast Cancer & 43 & 25.4 & 1 & 1 & 2 & $\mathrm{SLN}+(<10 \mathrm{~mm})$ \\
\hline & & & & Total $=23$ & Total $=\mathbf{3 1}$ & Total $=29$ & \\
\hline
\end{tabular}

Abbreviations: NA: not available; BMI: body mass index; SLNs: sentinel lymph nodes.

Table 7. SPECT/CT Versus Planar Imaging for Sentinel Lymph Node Detection

\begin{tabular}{|c|l|l|l|}
\hline Patients & \multicolumn{1}{|c|}{ Primary Tumour Sites } & \multicolumn{1}{|c|}{ SLN Sites on Planar Imaging } & \multicolumn{1}{|c|}{ SLN Sites on SPECT/CT } \\
\hline \hline 1 & Penile Cancer (penis - post penilectomy) & 2 inguinal & 2 superficial inguinal + 1 deep ilio-femoral \\
\hline 2 & Malignant Melanoma (left knee) & 1 inguinal & 1 superficial inguinal + 1 deep ilio-femoral \\
\hline 3 & Breast Cancer (left breast) & 1 left axilla & 3 left axilla : levels I / II / III \\
\hline 4 & Malignant Melanoma (left abdomen) & 1 left axilla +1 left chest & 1 left axilla +1 left chest wall (subcutaneous) \\
\hline 5 & Malignant Melanoma (back) & 1 right axilla +3 right chest & 1 right axilla +3 right intercostal \\
\hline 6 & Malignant Melanoma (midline abdomen) & $\begin{array}{l}1 \text { right axilla }+3 \text { left axilla }+1 \text { equivocal } \\
\text { focus in the abdomen }\end{array}$ & 1 right axilla +3 left axilla +1 mesenteric \\
\hline 7 & Breast Cancer (left breast) & 2 left axilla & 2 left axilla : levels I and II (interpectoral) \\
\hline 8 & Breast Cancer (right breast) & $\begin{array}{l}1 \text { right axilla }+1 \text { right extra-axillary in the } \\
\text { chest }\end{array}$ & 1 right axilla (level I) + 1 right internal mammary \\
\hline 9 & Breast Cancer (right breast) & 1 right axilla & 1 right axilla $:$ level I \\
\hline 10 & Breast Cancer (left breast) & 1 left axilla & 4 left axilla $:$ levels I and II \\
\hline 11 & Breast Cancer (right breast) & 1 right axilla & 2 right axilla : levels I and II \\
\hline 12 & Breast Cancer (left breast) & 1 left axilla & 1 left axilla : level I \\
\hline
\end{tabular}

planar imaging. SPECT/CT was found useful for anatomic localisation of disease extent per abdominal region, and also for differentiation between intraluminal uptake (i.e. free ${ }^{99 \mathrm{~m}} \mathrm{Tc}$ or ${ }^{99 \mathrm{~m}} \mathrm{Tc}-\mathrm{WBC}$ ) and active CD (i.e. colon wall uptake). In $11(47.8 \%)$ patients with active CD, low-dose multislice CT revealed bowel wall thickening, lumen narrowing, entero-cutaneous fistula, rectal abscess, ileostomy, small bowel herniation, and surgical clips (Table 10).

\section{Parathyroid Studies (PTH)}

In the 42 consecutively operated patients with hyperparathyroidism, delayed SPECT/CT imaging ( $2 \mathrm{~h}$ posti.v.) significantly improved the mean sensitivity $(63.2 \% \mathrm{vs}$ $88.2 \% ; \mathrm{p}<0.01)$ and the mean diagnostic accuracy $(64.7 \% \mathrm{vs}$
$83.8 \% ; \mathrm{p}<0.05)$ compared to ${ }^{99 \mathrm{~m}}$ Tc-MIBI double-phase planar imaging $(10 \mathrm{~min}$ and $2 \mathrm{~h}$ post-i.v.) for detection of adenomas and hyperplasia. Compared to surgical and pathological findings, SPECT/CT had $88.6 \%$ sensitivity, $97.4 \%$ specificity, $95.4 \%$ diagnostic accuracy, $96.6 \%$ negative predictive value, and $91.2 \%$ positive predictive value for accurate anatomic localisation of solitary parathyroid adenomas. Ectopic P3 and P4 adenomas were accurately localised to the thyrothymic tract, the tracheoesophageal groove, the retrotracheal region, the retroclavicular region, the thyroid gland, the carotid sheath, the aorto-pulmonary window, and the pericardic fat (see Fig. 5). 
Table 8. ProstaScint SPECT/CT : Added-Value of SPECT/CT versus Planar/SPECT

\begin{tabular}{|c|c|c|c|c|c|c|}
\hline Indications & PSA Levels (ng/ml) & Planar WBS & SPECT/CT & $\begin{array}{l}\text { Impact on } \\
\text { TRT }\end{array}$ & $\begin{array}{c}\text { Treatment } \\
\text { Administered }\end{array}$ & $\begin{array}{c}\text { Added-Value of SPECT/CT } v s \\
\text { Planar/SPECT }\end{array}$ \\
\hline SR & 2.89 & NED & PLD & Yes & HT & \multirow{25}{*}{$\begin{array}{l}\text { 1- Planar WB : } \\
\text { Useful for detection of EPDD but } \\
\text { limited for detection of LPD } \\
\text { 2- SPECT : } \\
\text { Useful for detection of small pelvic } \\
\text { foci but blinded to anatomic } \\
\text { localisation } \\
\text { 3- SPECT/CT : } \\
\text { - Improved confidence for } \\
\quad \text { image interpretation based on } \\
\text { anatomy and attenuation } \\
\text { correction } \\
\text { Improved sensitivity, } \\
\text { especially for detection of } \\
\text { small pelvic recurrence } \\
\text { Possible impact on } \\
\text { management even in patients } \\
\text { with slow rise of PSA }\end{array}$} \\
\hline SR & 0.3 & NED & NED & No & pXRT & \\
\hline SR & 6.72 & NED & PLD & Yes & pXRT & \\
\hline SR & 0.42 & NED & PLD & Yes & pXRT & \\
\hline SW & $<0.1$ & NED & PLD & Yes & pXRT & \\
\hline SR & 1.05 & NED & PLD & Yes & pXRT & \\
\hline SR & 0.24 & NED & PLD & Yes & pXRT & \\
\hline SR & 0.71 & NED & NED & Yes & HT & \\
\hline SR & 6.22 & NED & PLD & No & Clinical Trial $*$ & \\
\hline SR & 0.78 & NED & PLD & Yes & pXRT & \\
\hline SR & 2.4 & NED & PLD & Yes & HT & \\
\hline IS & 9.1 & NED & PLD & No & $\mathrm{XRT}+\mathrm{HT}$ & \\
\hline SR & 2.1 & NED & PLD & Yes & pXRT & \\
\hline SR & 0.28 & NED & PLD & Yes & pXRT & \\
\hline SW & $<0.1$ & NED & PLD & No & $\mathrm{HT}$ & \\
\hline SR & 3.8 & NED & EPLD & Yes & HT & \\
\hline SR & 3.37 & NED & NED & Yes & pXRT & \\
\hline SR & 15.2 & NED & PLD + EPDD & Yes & pXRT & \\
\hline SR & 14 & NED & PLD + EPDD & Yes & HT & \\
\hline SW & $<0.1$ & NED & PLD & Yes & pXRT & \\
\hline SR & 6.5 & NED & NED & No & Surveillance & \\
\hline SR & 2.17 & EPDD & PLD + EPDD & Yes & Surveillance & \\
\hline SW & $<0.04$ & NED & EPLD & Yes & HT & \\
\hline SR & 0.33 & NED & PLD + EPDD & Yes & Surveillance & \\
\hline SR & 9.56 & EPDD & PLD + EPDD & Yes & HT & \\
\hline SR & 0.15 & NED & NED & Yes & Surveillance & \\
\hline SR & 20.1 & NED & NED & No & HT & \\
\hline
\end{tabular}

Abbreviations: WBS: whole-body scan; TRT: treatment; SR: suspicion of recurrence; IS: initial staging; SW: salvage therapy; NED: no evidence of disease; PLD: pelvic local disease; EPDD: extra-pelvic distant disease; HT: hormone therapy; pXRT: pelvic external radiation therapy; Clinical trial: Denosumab ${ }^{\circledR}$ protocol. $^{\circ}$

Table 9. Added-Value of the Infinia-Hawkeye -4 in ${ }^{99 m}$ Tc-Red Blood Cell Imaging

\begin{tabular}{|c|c|c|c|}
\hline Patients & Indications & Conventional Imaging Work-Up & SPECT/CT Versus Planar/SPECT \\
\hline 1 & Liver hemangioma & ND & \multirow{11}{*}{$\begin{array}{l}\text { - Improved interpretation confidence } \\
\text { - Differentiation between physiological uptakes (aorta, } \\
\text { intrahepatic vessels) versus pathological uptakes } \\
\text { (hemangiomas, splenic functional remnants) } \\
\text { - } \quad \begin{array}{l}\text { Better anatomic localisation of liver and splenic lesions, as } \\
\text { well as abdominal nodules }\end{array} \\
\text { - } \quad \begin{array}{l}\text { Detection of morphological lesions but suboptimal for } \\
\text { small liver lesions }(<1.5 \mathrm{~cm})\end{array} \\
\text { - } \quad \text { Faster or final diagnosis. Further work-up avoided. }\end{array}$} \\
\hline 2 & Liver hemangioma & FD-CT, HR-MRI & \\
\hline 3 & Splenosis & $\mathrm{CT}$ & \\
\hline 4 & Liver hemangioma & US & \\
\hline 5 & Liver hemangioma & FD-CT & \\
\hline 6 & Spleen hemangioma & FD-CT, US & \\
\hline 7 & Splenosis & US & \\
\hline 8 & Liver hemangioma & US & \\
\hline 9 & Liver hemangioma & US & \\
\hline 10 & Liver hemangioma & FD-CT & \\
\hline 11 & Liver hemangioma & FD-CT & \\
\hline
\end{tabular}




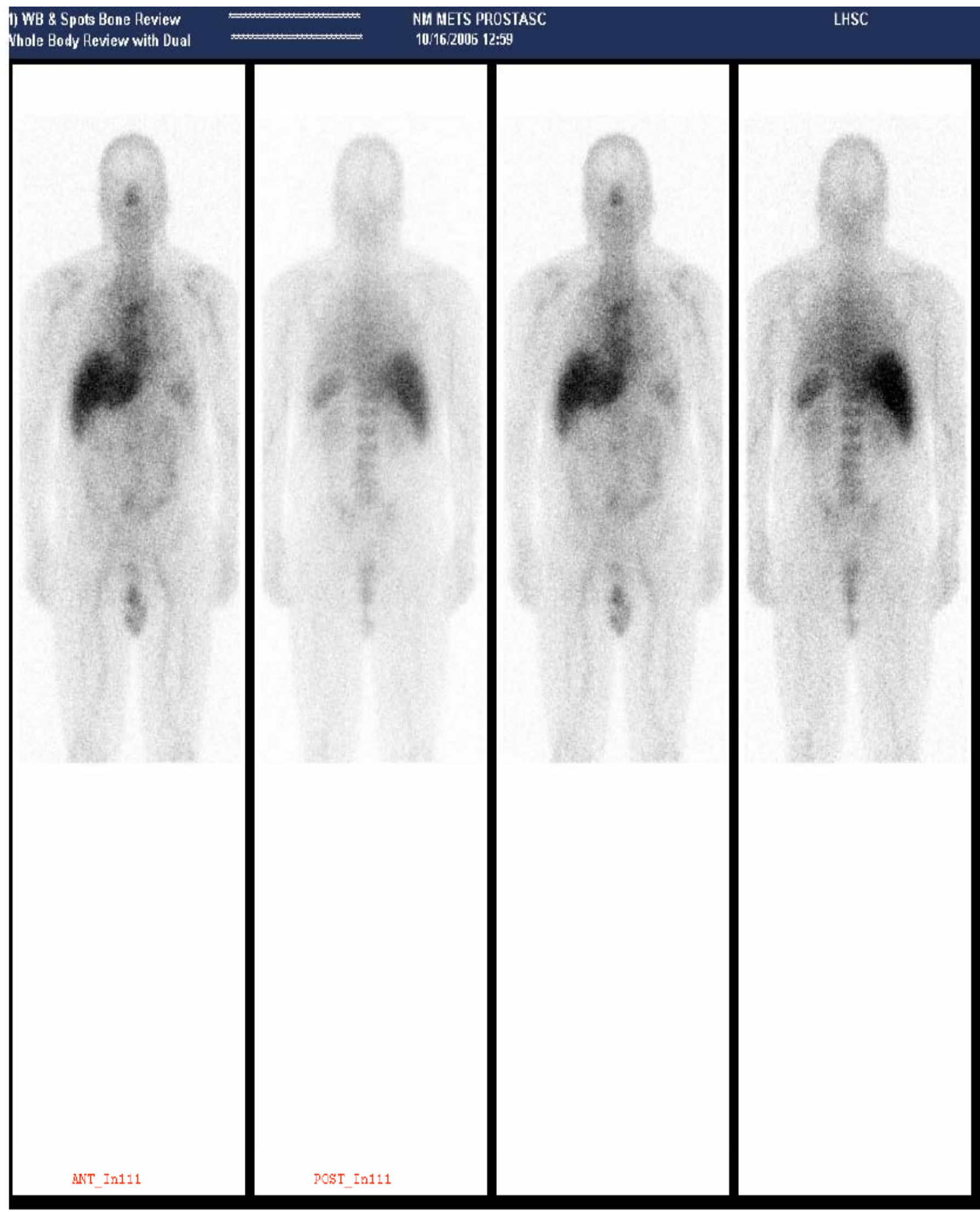




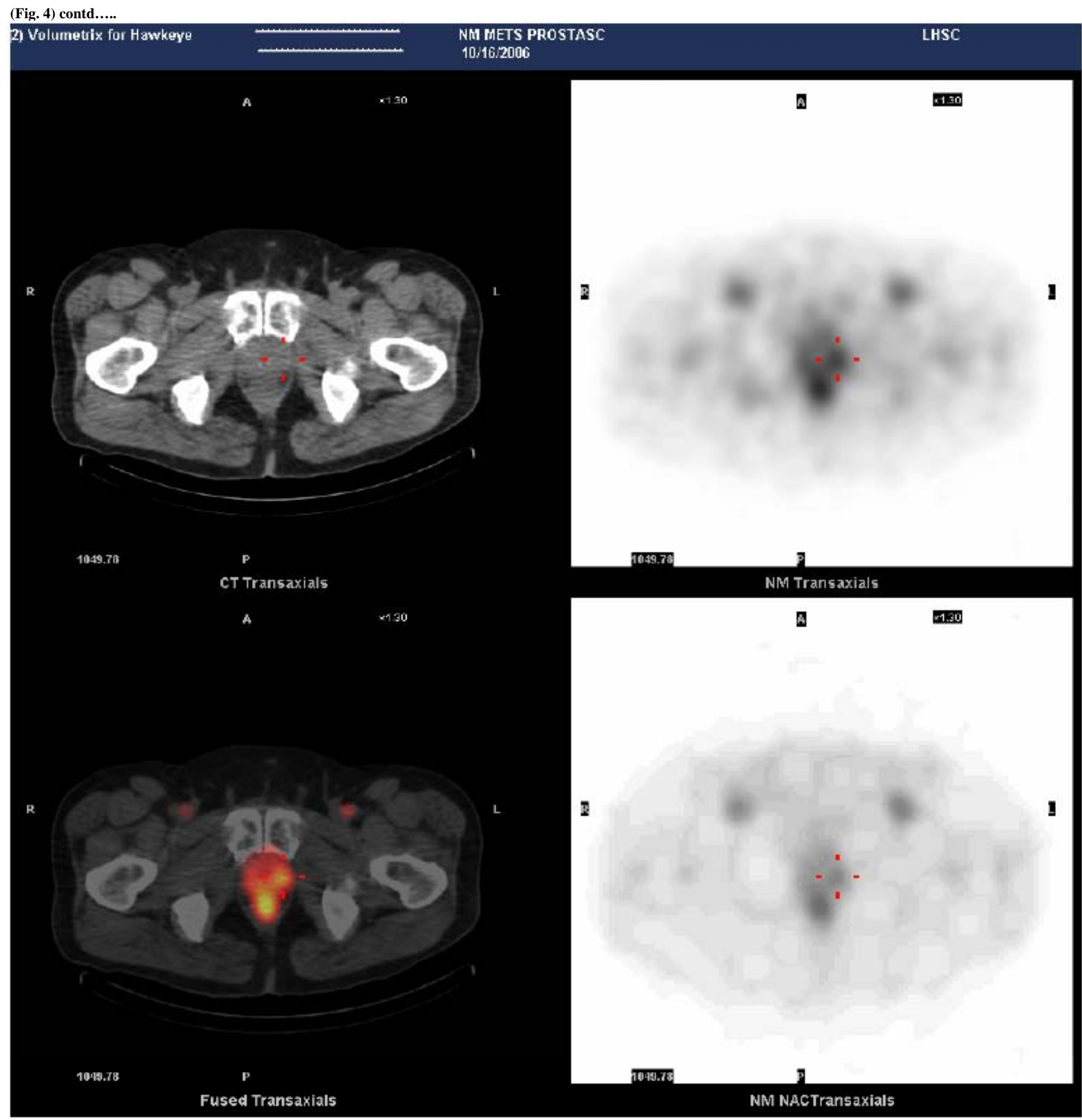

Fig. (4). A 61-year old male with a prostate cancer (cT3aNx, Gleason 7) was evaluated with planar whole-body scan (WBS) and SPECT/CT ProstaScint ${ }^{\circledR}$ after rise of serum PSA levels $(2.4 \mathrm{ng} / \mathrm{ml})$ following an external radiation therapy. Planar WBS showed normal tracer distribution. Integrated SPECT/low-dose multislice CT revealed a small focus in the left prostate bed, which was strongly suggestive of local pelvic disease.

SPECT/low-dose multislice CT was successfully incorporated into the surgical planning for precise 3D localisation of abnormal parathyroid glands, resulting in a significant reduction in operating and post-operative recovery times. In $70 \%$ of these patients with a primary hyperparythoidism de novo, image-guide surgery led to a unilateral neck parathyroidectomy with normalization of
iPTH/Ca levels post-operatively; no laryngeal nerve palsy or severe hypocalcaemia complications were noted except five minor hypocalcaemia at the lower normal range (Table 11).

\section{Brain Studies}

In five patients with multifocal glioma $(n=1)$ or oligodendroglioma $(n=4)$, and two patients with brain 
Table 10. Added-Value of Infinia-Hawkeye -4 in ${ }^{99} \mathrm{~m}$ Tc-HMPAO-WBC Imaging

\begin{tabular}{|c|c|}
\hline Indications & Added-Value of SPECT/CT Versus Planar/SPECT \\
\hline $\begin{array}{l}\text { Crohn's Disease } \\
\quad(\mathbf{n}=\mathbf{2 3})\end{array}$ & 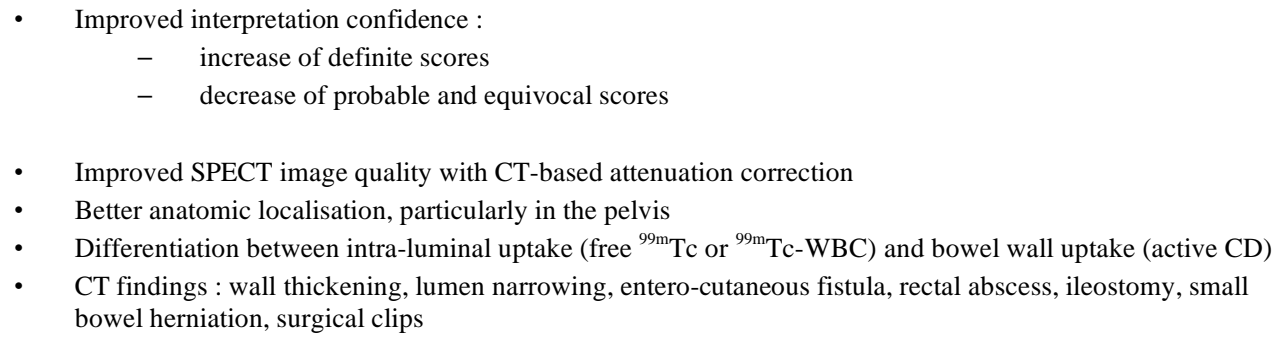 \\
\hline $\begin{array}{l}\text { Suspicion of Osteomyelitis } \\
\qquad(\mathbf{n}=15)\end{array}$ & $\begin{array}{l}\text { - Improved interpretation confidence : } \\
-\quad \text { increase of definite scores } \\
-\quad \text { decrease of probable and equivocal scores } \\
\text { - Differentiation between soft-tissue infection and osteomyelitis } \\
\text { - Better anatomic localisation }\end{array}$ \\
\hline
\end{tabular}

metastases from a breast cancer $(n=1)$, and a renal cell cancer $(n=1)$, nine SPECT/CT studies were performed with ${ }^{99 \mathrm{~m}}$ Tc-MIBI $(\mathrm{n}=3)$ and/or ${ }^{201} \mathrm{Tl}(\mathrm{n}=6)$ after surgery and/or chemotherapy and/or radiation therapy to differentiate recurrence from necrosis on brain MRI or fully-diagnostic contrast-enhanced CT. Fused images were found useful for accurate anatomic and functional localisation of tumour recurrence, although the contrast-resolution of the low-dose unenhanced CT from SPECT/CT was found inherently insufficient (Table 12). Software image fusion between SPECT/low-dose multislice CT and high-resolution gadolinium-enhanced MRI was routinely performed for more appropriate interpretation.

\section{Clinical Research}

In the 9 oncology patients with lymphomatous masses $(n=4)$ treated by ${ }^{131}$ I-tositumomab (Bexxar $\left.{ }^{\circledR}\right)$ and liver metastases from carcinoids $(n=5)$ treated by ${ }^{131}$ I-Lipiodol (Lipiocis $^{\circledR}$ ), the 4 slice SPECT/CT system was found of additional value for anatomic and functional characterisation of tracer distribution, especially in heterogeneous necrotic tumours
Table 11. Added-Value of Infinia-Hawkeye 4 slice in Parathyroid Imaging

Planar Plus SPECT/CT Guided Surgical Planning : Surgeons' Feed Back

- Routine protocol for image-guided surgery

- Accurate 3D anatomic localisation of SPA

- Reduction in operating time

- Reduction in recovery time

- Unilateral parathyroidectomy in $70 \%$ of patients with SPA

- SPECT/CT based MIRS in well selected patients

Abbreviations: SPA: solitary parathyroid adenoma; MIRS: minimally invasive radioguided surgery.

(Fig. 6). In the 4 patients with benign multinodular goiters $(n=3)$ and Graves' disease $(n=1)$, SPECT/CT was used for calculation of ${ }^{131}$ I uptake and definition of anato-functional volume before ${ }^{131} \mathrm{I}$ therapy. In the 33 cardiology patients, CT-based attenuation correction was used as a pathway

Table 12. Added-Value of Infinia-Hawkeye 4 Slice in Brain Imaging

\begin{tabular}{|c|c|c|c|c|}
\hline Brain Scans & Primary Tumour Histology & Tracer & Results & SPECT/CT Versus SPECT \\
\hline 1 & Multifocal Glioma (Low Grade) & MIBI & Recurrence & \multirow{9}{*}{$\begin{array}{l}\text { - Improved image quality } \\
\text { - Increase AC/NAC counts ratio after attenuation } \\
\text { correction } \\
\text { - Anatomic localisation of post-surgery cavity, post- } \\
\text { radiation edema, necrotic mass, tumour recurrence }\end{array}$} \\
\hline 2 & Anaplastic Oligodendroglioma (High Grade) & MIBI & Recurrence & \\
\hline 3 & Anaplastic Oligodendroglioma (High Grade) & Tl-201 & Recurrence & \\
\hline 4 & Metastases (Breast Cancer) & $\mathrm{Tl}-201$ & Recurrence & \\
\hline 5 & Metastases (Renal Cancer) & MIBI & Recurrence & \\
\hline 6 & Metastases (Renal Cancer) & Tl-201 & Recurrence & \\
\hline 7 & Anaplastic Oligodendroglioma & Tl-201 & Recurrence & \\
\hline 8 & Oligodendroglioma (Intermediate Grade) & Tl-201 & Recurrence & \\
\hline 9 & Anaplastic Oligodendroglioma & Tl-201 & Recurrence & \\
\hline
\end{tabular}



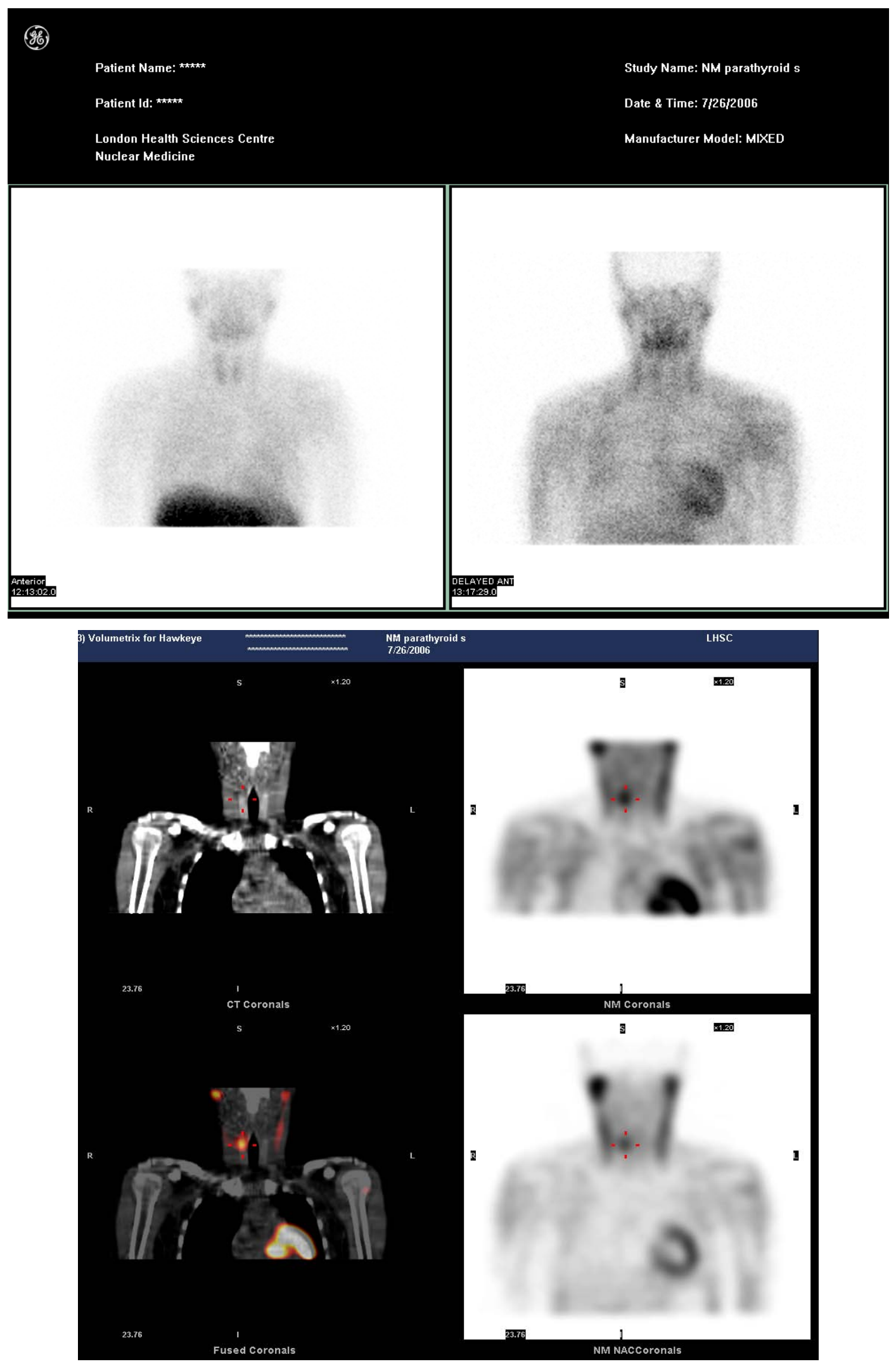

Fig. (5). A 16-year old female presented with a primary hyperparathyroidism. Ultrasound of the thyroid showed two solid hypoechoic structures adjacent to the upper and lower right thyroid lobe. A double-phase planar 99mTc-MIBI scan showed an equivocal focus in the right upper lobe of the thyroid, which was definitely positive on SPECT/low-dose multislice CT. After a right parathyroidectomy, a P4 parathyroid adenoma $(2.0 \times 0.8 \times 0.7 \mathrm{~cm})$ was confirmed at the final pathology. No other adenoma was identified in the lower right lobe. $\mathrm{iPTH} / \mathrm{Ca}$ serum levels were normalised post-surgery. 


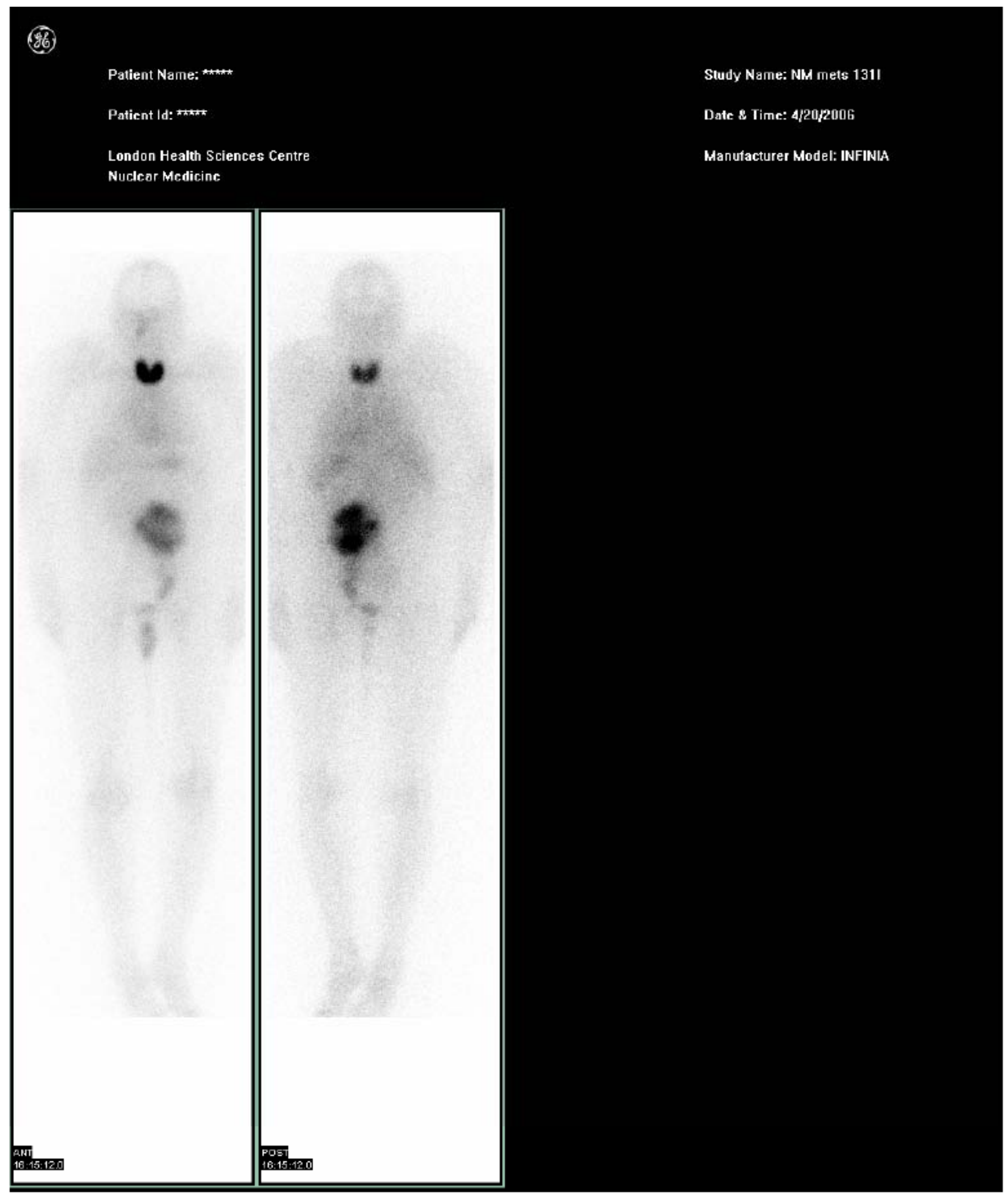


(Fig. 6) contd....

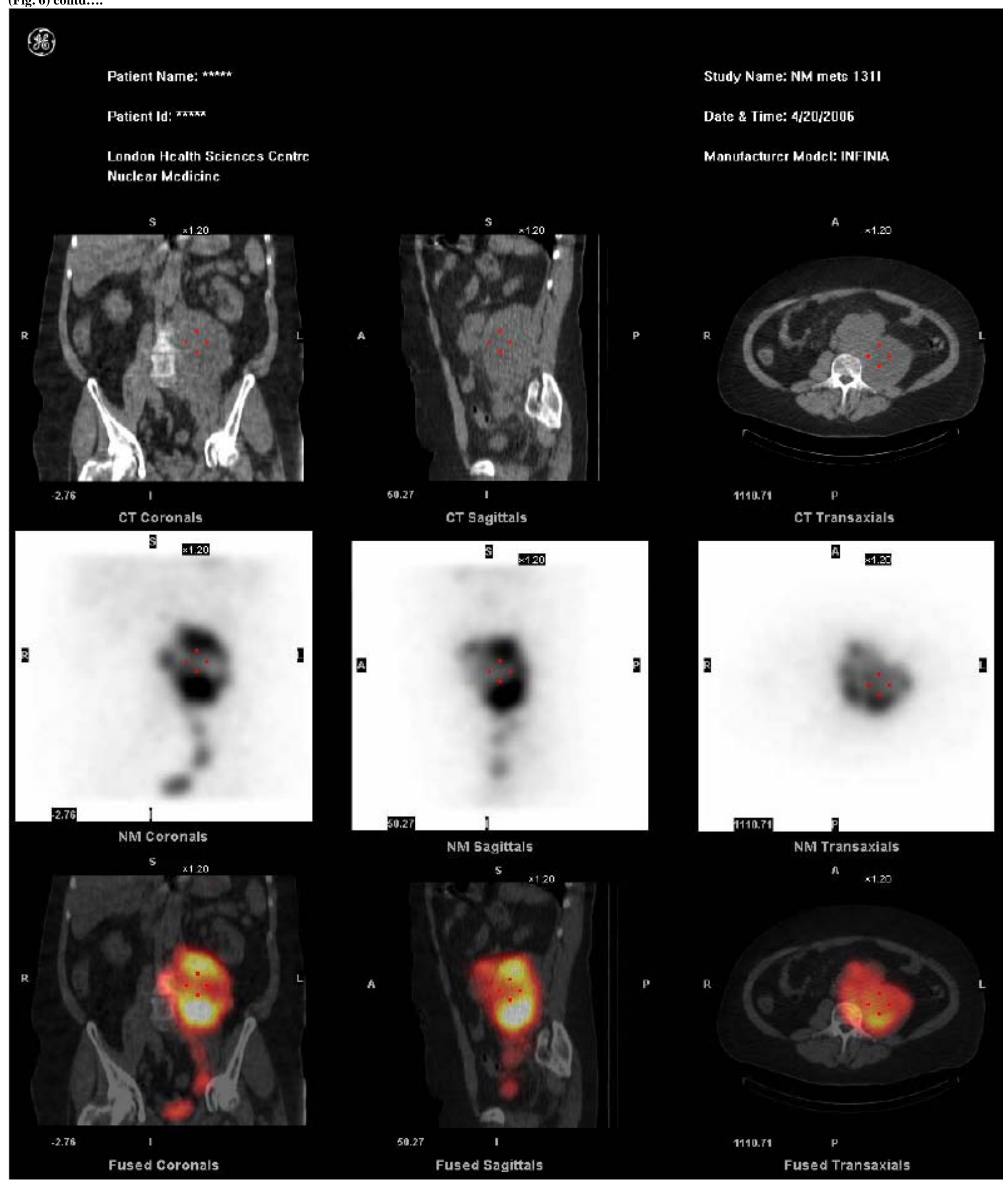

Fig. (6). A 57-year old patient with a non-Hodgkin lymphoma underwent a radioimmunotherapy with ${ }^{131}$ I-tositumomab $\left(\right.$ Bexxar $\left.{ }^{\circledR}\right)$. The administered dose $(4.9 \mathrm{GBq})$ was based on planar whole-body imaging (left). The patient experienced a few weeks of dramatic symptom relief followed by a rapid recurrence. Note on the SPECT image, the CT image, and fused SPECT/CT images, respectively, the heterogeneous distribution of ${ }^{131} \mathrm{I}$-tositumomab throughout the tumour mass overlapping the left body vertebra of L3 (right). The recurrence may have been attributable to the regional variation in delivered radiation dose. SPECT/low-dose multislice CT may be used as a research tool for 4D (3D+time) tumour dosimetry. 
towards half-time scan with resolution recovery. Accordingly, in a half-time acquisition, fully corrected SPECT/CT with resolution recovery offers the potential to significantly improve the image quality, while maintaining the same diagnostic accuracy than full-time SPECT/CT for detection of coronary artery disease.

Table 13 summarizes the added-value of the Infinia ${ }^{\mathrm{TM}}$ Hawkeye $^{\mathrm{TM}} 4$ slice per clinical indication.

Table 14 summarizes the added-value of the Infinia ${ }^{\mathrm{TM}}$ Hawkeye ${ }^{\mathrm{TM}} 4$ slice in research.

\section{DISCUSSION}

SPECT/CT is playing an incremental role in nuclear imaging $[2,20,21]$. In comparison with PET/CT, however, the optimal positioning of SPECT/CT has still to be defined for appropriate patient management [21-28]. We wanted to answer a key-question: how useful is an integrated SPECT/low-dose multislice CT in clinical setting and research? This interrogation is warranted because SPECT/CT models vary widely in terms of the CT sophistication and the contribution to interpretation of the hybrid images requires evaluation $[4,26,29,30]$.

Hybrid cameras currently available on the market may be classified in terms of low dose $v s$ high dose, single slice $v s$ multislice, slow-rotating $v s$ fast-rotating, or low contrast- resolution $v s$ high contrast-resolution depending on the CT specifications [9, 31, 32]. In a series of 81 consecutive patients, Schillaci et al. found a SPECT/low-dose lowresolution CT (Millennium VG \& Hawkeye, GE Healthcare) useful to improve scintigraphic imaging interpretation. In this reference prospective study, SPECT/CT improved the diagnostic accuracy compared to conventional planar/SPECT imaging, especially for anatomic localisation of pathological uptake, definition of functional significance of CT lesions, and exclusion of disease in sites of physiological uptake [5]. In a recent article, Jacene et al. reported their two year clinical experience with a SPECT/low-dose single-slice CT (Millennium VG \& Infinia Hawkeye -1, GE Healthcare) in 144 patients referred for various non-cardiac indications. These authors showed the added-value of SPECT/CT over SPECT alone for improving the interpretation confidence with more definitive anatomic localisation and more definitive diagnostic certainty [33]. Similarly, Bailey and al. developed a SPECT/low-dose single-slice CT scanner (SKYlight/Picker PQ 5000, Philips Medical System). In the first year of operation in more than 200 patients, this modular SPECT/CT system was found cost-effective with the possibility to use the low-cost high spatial resolution spiral CT unit as a diagnostic CT with an added-value of SPECT/CT over planar/SPECT alone [29, 34]. Buck and al. recently reported their experience

Table 13. Clinical Added-Value of the Infinia ${ }^{\mathrm{TM}}$ Hawkeye $^{\mathrm{TM}} 4$ Slice

\begin{tabular}{|c|c|}
\hline Clinical Indications & Added-Value of The Infinia ${ }^{\mathrm{TM}}$ Hawkeye $\mathrm{T}^{\mathrm{TM}} 4$ Slice \\
\hline MPI $(n=70)$ & $\begin{array}{l}\left.\text { Increase of normalcy rates and specificity regardless to the reader's experience, especially in obese patients (BMI } \geq 30 \mathrm{~kg} / \mathrm{m}^{2}\right) \\
\text { Availability of software quality control for correction of misalignment - Feasibility of } 1 \mathrm{~mA} \text { CT acquisition for accurate } \\
\text { attenuation correction }\end{array}$ \\
\hline${ }^{131} I(n=42)$ & $\begin{array}{l}53 \% \text { of pts: better charaterisation of equivocal foci on planar WBS related to physiological or benign }{ }^{131} \mathrm{I} \text { uptakes } \\
33 \% \text { of pts: better anatomic localisation of }{ }^{131} \mathrm{I} \text {-avid metastases - Detection of CT abnormalities related to nodal, brain, bony, and } \\
\text { lung metastases }\end{array}$ \\
\hline $\operatorname{SLN}(n=12)$ & $\begin{array}{l}\text { Detection of hot SLNs not seen on planar imaging including deep nodes or hot nodes hidden by scattered radiation } \\
\text { Precise anatomic localisation of SLNs per nodal station - Detection of }\langle 1 \mathrm{~cm} \text { or }>1 \mathrm{~cm} \text { hot SLNs on low-dose multislice CT }\end{array}$ \\
\hline $\operatorname{ProstaScint}^{\circledR}(n=27)$ & $\begin{array}{l}100 \% \text { of pts : improved interpretation confidence compared to planar WBS/SPECT } \\
\text { Differentiation between physiological uptakes (gut, vessels, bone marrow) and pathological uptakes (local and/or distant } \\
\text { recurrence) } \\
\text { Improved detection of small pelvic recurrence in patients with normal CT/MRI/Bone scan and elevated PSA } \\
\text { Detection of CT abnormalities (lymph nodes, Paget disease, degenerative disease, bladder wall thickening) }\end{array}$ \\
\hline PTH $(n=42)$ & $\begin{array}{l}\text { Improved interpretation confidence compared to planar dual-phase imaging } \\
\text { Significant increase of sensitivity }(\mathrm{p}<0.01) \text {, and diagnostic accuracy }(\mathrm{p}<0.05) \text { for detection of SPA } \\
\text { High specificity for 3D-anatomic localisation of eutopic and ectopic SA with a significant impact on surgical planning }\end{array}$ \\
\hline Bone scan $(n=51)$ & $\begin{array}{l}\text { 82\% of oncology pts : SPECT/CT allowed final or faster diagnosis in patients with an equivocal planar WBS } \\
37 \% \text { of pts : SPECT/CT allowed guidance of appropriate work-ups (HR-MRI or FD-CT) } \\
75 \% \text { of oncology pts : Evolution allowed to cover the entire spine in less than } 30 \text { min with improved IQ vs OSEM vs FBP } \\
92 \% \text { of pts : CT quality was found good or acceptable for detection of spine degenerative disease (osteophytes, disk narrowing, } \\
\text { facet joint arthritis) vs osteolytic/blastic metastases }\end{array}$ \\
\hline Brain $(n=7)$ & Accurate anatomic and functional localisation of hot spots related to tumour recurrence \\
\hline $\mathbf{R B C}(\mathbf{n}=11)$ & $\begin{array}{l}\text { Accurate anatomic localisation of hot spots related to liver or spleen hemangiomas as well as of functional splenic remnants or } \\
\text { splenosis }\end{array}$ \\
\hline WBC $(n=38)$ & $\begin{array}{l}\text { Accurate diagnosis of osteomyelitis } v s \text { soft tissue infection } \\
\text { Improved interpretation confidence for diagnosis or exclusion of active Crohn's disease compared to planar imaging } \\
\text { CT findings in active CD (wall thickening, lumen narrowing, fistulae, rectal abscess, ileostomy, herniation, surgical clips) }\end{array}$ \\
\hline${ }^{67} \mathbf{G a}(\mathrm{n}=40)$ & $\begin{array}{l}58 \% \text { of pts : Incremental value of SPECT/CT compared to planar WBS and SPECT alone, especially for better anatomic } \\
\text { localisation, faster or final diagnosis, and differentiation between physiological versus pathological uptakes } \\
48 \% \text { Detection of CT abnormalities (lung, lymph nodes, bone marrow, liver, spleen) }\end{array}$ \\
\hline $\begin{array}{l}\text { 131/123 I-MIBG }(n=19) \\
\text { Octreoscan }^{\circledR}(n=51)\end{array}$ & $\begin{array}{l}\text { Improved confidence for interpretation with more accurate localisation of disease and exclusion of physiological uptakes } \\
\text { Detection of CT abnormalities, which were unsuspected on WB and SPECT (i.e. lung metastases) }\end{array}$ \\
\hline
\end{tabular}

Abbreviations: WBS: whole-body scan; AC: attenuation correction; QC: quality control; HR-MRI: high-resolution MRI, VCT: volumetric CT; SPA: single parathyroid adenomas; FD-CT: fully diagnostic CT; SW: software; LN: lymph node; DTC: differentiated thyroid cancer; PSA: prostate specific antigen. 
with a SPECT/multislice CT scanner (Symbia T6, Siemens). In non-cardiac protocols, these authors highlighted the superiority of SPECT/CT over planar/SPECT with a CT unit that can be used as a low-dose non-enhanced spiral CT or high-dose contrast-enhanced diagnostic CT. In cardiac protocols, low-dose non-enhanced CT may be used for attenuation correction in MPI or combined to a diagnostic 64slice cardiac CT for coronary angiography and calcium scoring [35].

In London hospitals, we are building a hybrid imaging platform incorporating five SPECT/CT (two Infinia-Hawkeye 1, two Infinia-Hawke -4, and one Symbia T6), in addition to one 64-slice VCT and one 64-slice PET/CT. Our pilot experience in 456 patients evaluated on the Infinia ${ }^{\mathrm{TM}}$ Hawkeye $^{\mathrm{TM}} 4$ slice (HWK-4, GE Healthcare) indicates that SPECT/low-dose multislice $\mathrm{CT}$ is useful in a large spectrum of clinical indications. SPECT/CT is currently implemented worldwide for clinical applications, especially for ${ }^{111}$ In-Octreotide $\left(\right.$ Octreoscan $\left.^{\circledR}\right)$, ${ }^{131 / 123} \mathrm{I}-\mathrm{MIBG},{ }^{131} \mathrm{I},{ }^{67} \mathrm{Ga}$, and ${ }^{111}$ In-Capromab Pendetide $\left(\right.$ ProstaScint $\left.^{\circledR}\right)$ studies [20, 29, 36, 37]. For these oncological indications using medium-energy and high-energy isotopes, accurate 3D localisation of ill-defined hot-spots is critical. Lowdose SPECT/CT significantly improved the confidence in the interpretation compared to planar views and SPECT alone. By definition, the strength of low-dose SPECT/CT is the low CT radiation exposure $(<2 \mathrm{mSv})$, which is similar to the yearly natural background $(\sim 3 \mathrm{mSv})$. From a clinical point of view, the third generation CT was able to detect gross morphological abnormalities including nodal, lung, pleural, brain, bone, spleen, and liver metastases with sufficient contrast-resolution to allow either a final diagnosis or an appropriate guidance of further investigations. Importantly, low-dose multislice CT from integrated SPECT/CT was not designed to substitute for a fullydiagnostic CT but to primarily improve the SPECT capabilities [38]. Hence, no contrast agent enhancement was used in our initial experience, although this option might be considered for particular abdominal and pelvic indications. CT training for the nuclear medicine physician appears indispensable to take advantage of the CT added-value in clinical routine. Respiratory motion artifacts located at the lung-liver interface was the main limitation for the optimal detection of small liver lesions. In comparison to the Infinia-Hawkeye -1 (HWK -1), the CT scan time was shortened by half (5 min versus $10 \mathrm{~min}$ ), which significantly improved the patient's compliance. Also, the helical acquisition from HWK -4 with a $5 \mathrm{~mm}$ slice thickness significantly improved the contrast-resolution with fewer motion and step-stairs artifacts compared to the axial acquisition from HWK -1 with a $10 \mathrm{~mm}$ slice thickness. ACQC for MPI was available on HWK $-4 /$ Xeleris $2.05 \mathrm{v}$, compared to the HWK $1 /$ Xeleris $1.1 \mathrm{v}$, thereby, reducing misalignment-generated artifacts: our MPIs are routinely performed with SPECT/CT and processed with ACQC for every single patient, which significantly improved the diagnostic accuracy [39]. In contrast to high-dose/-resolution SPECT/CT models, a possible weakness of the Hawkeye -4 unit is the lack of functionality as a standalone CT study. As a slow-rotating/low-dose SPECT/CT system, the scan time duration from HWK-4 considerably reduced the patient throughput. In these indications, a faster CT may eventually improve both the image quality and patient's compliance for the daily routine. For whole-body assessment of oncology patients, ${ }^{18} \mathrm{FDG}$, and new PET tracers such as ${ }^{18} \mathrm{~F}-$ fluoro-L-thymidine $\left({ }^{18} \mathrm{FLT}\right),{ }^{18} \mathrm{~F}$-fluoro-methyl-choline $\left({ }^{18} \mathrm{FCH}\right)$, or ${ }^{68}$ Ga-radiolabelled peptides, renders the need for SPECT/CT quite limited to departments where PET/CT is available. In our practice, high-resolution MRI is the modality of choice for diagnosing brain lesions. SPECT/CT-MRI software image fusion was used due to the insufficient contrast-resolution of the low-dose multislice CT from our SPECT/CT.

For non-oncological indications such as parathyroid, RBC and WBC studies, low-dose SPECT/CT was found useful for accurate 3D localisation of suspicious or equivocal findings: SPECT/CT is now routinely used for our parathyroid studies for surgical planning. For RBC studies, most patients were referred after non-specific findings with fully-diagnostic CT (FD-CT) or high-resolution MRI (HR-MRI); in the rare cases with small lesions, which are undetectable by low-dose SPECT/CT, SPECT-FDCT or SPECT-HRMRI software fusion might be superior. In patients referred for bone scans, low-dose SPECT/CT with resolution recovery (i.e. Bone Evolution) significantly improved the image quality compared to FBP and OSEM reconstructions. Moreover, Bone Evolution is routinely implemented for half-time acquisition, which proved to be particularly useful in oncology patients who may require more than one FOV to clarify equivocal distant foci on the spine. Interestingly, SLN detection was another indication for which the low-dose SPECT/CT was found of clinical interest. This holds true not only for precise anatomic localisation of SLN nodes but also for detection of additional relevant hot lymph nodes, especially after attenuation correction; accordingly, SPECT/CT has the potential to re-visit the initial concept of SLN by defining anato-functional image patterns [40, 41].

In cardiology patients, HWK -4 provided accurate attenuation correction even with the lowest current $(1.0 \mathrm{~mA})$. A multireader evaluation showed the superiority of IRAC over IRNC with greater interpretation confidence, especially in overweight and obese patients with a probably normal or equivocal NC study.

Table 14. Added-Value of Infinia-Hawkeye 4 Slice in Research

\begin{tabular}{|c|c|}
\hline Oncology & Cardiology \\
\hline $\begin{array}{l}\text { Evaluation of IRT efficiency in patients treated by Bexxar }{ }^{\circledR} \text { and } \\
\text { Lipiocis } \\
\text { - Dosimetry calculations in benign MNG and Graves' disease based } \\
\text { on 3D anato-functional volume and patient's specific attenuation } \\
\text { maps } \\
\text { - Accurate absolute quantification of tracer uptake for }{ }^{99 \mathrm{~m}} \mathrm{Tc},{ }^{131} \mathrm{I},{ }^{123} \mathrm{I} \text {, } \\
\text { and }{ }^{111} \text { In isotopes } \\
\text { - Development of semi-quantitative SUV scores dedicated to SPECT- } \\
\text { CT imaging }\end{array}$ & $\begin{array}{ll}- & \text { Half-time resolution recovery : } \\
- & \text { Half-time SPECT/CT MPI (7.5 min) and CT-based AC, SC, and } \\
& \text { RR } \\
- & \text { Improved SPECT image quality } \\
- & \text { At least, similar diagnostic accuracy than routine full-time (15 min) } \\
& \text { SPECT }\end{array}$ \\
\hline
\end{tabular}

Abbreviations: SUV: standardised uptake value; AC: attenuation correction; SC: scatter correction; RR: resolution recovery. 
Table 15. Clinical Relevance of a SPECT/Low-Dose MultiSlice CT System

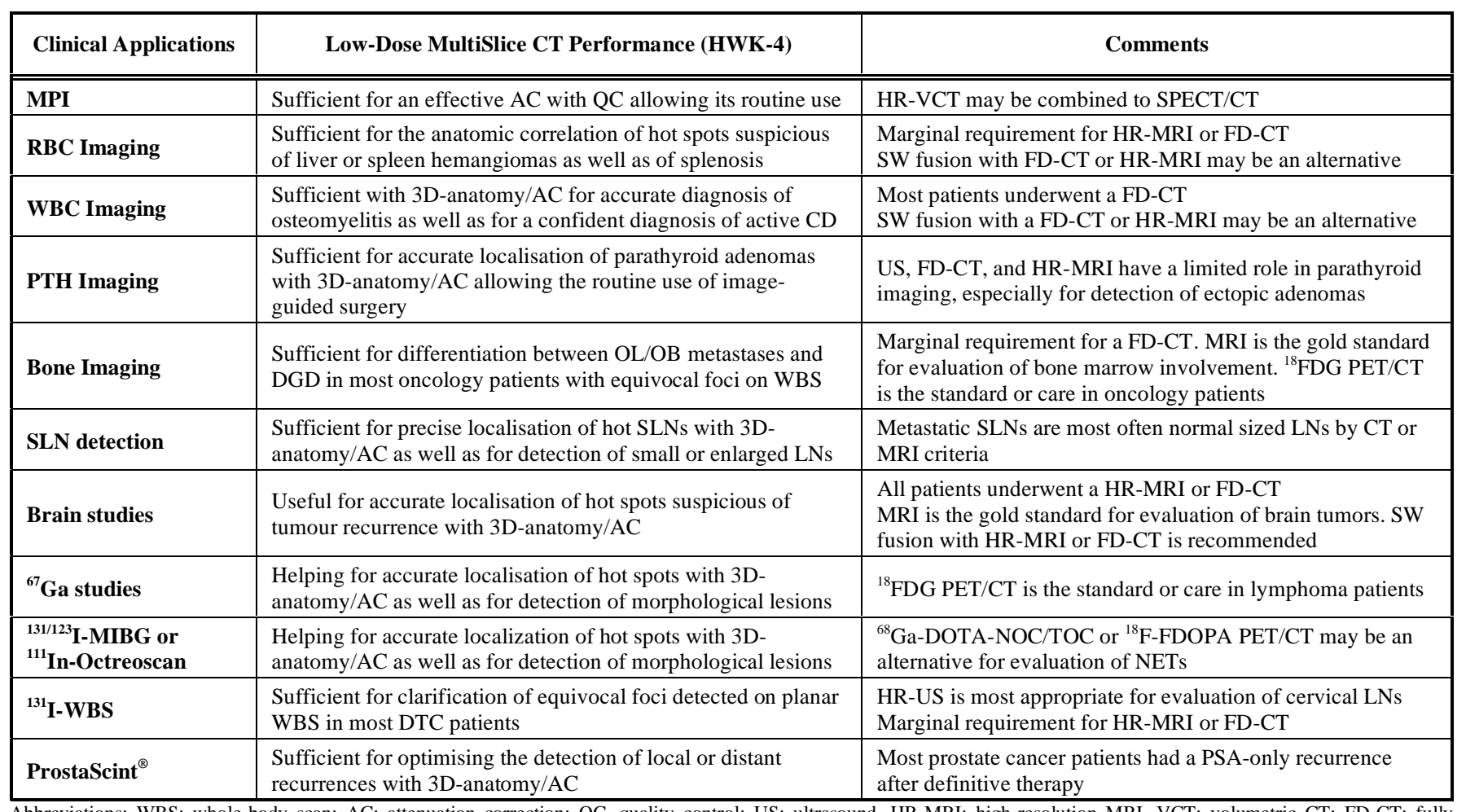

Abbreviations: WBS: whole-body scan; AC: attenuation correction; QC, quality control; US: ultrasound, HR-MRI: high-resolution MRI, VCT: volumetric CT; FD-CT: fully diagnostic CT; SW: software; LN: lymph node; DTC: differentiated thyroid cancer; DGD: degenerative disease; PSA: prostate specific antigen.

These findings are of clinical interest for minimising the radiation exposure, while maintaining or improving the specificity and diagnostic accuracy for detection of CAD.

In clinical research, low-dose SPECT/CT was used for evaluation of internal radiation therapy (IRT) efficiency in oncology patients treated by Bexxar ${ }^{\circledR}$. The tracer distribution may not necessarily match the anatomical extent of disease as observed in lymphomatous heterogeneous masses with a necrotic component. SPECT/CT was found promising immediately after Lipiocis ${ }^{\circledR}$ therapy, especially for confirmation of successful chemo-embolisation and exclusion of extra-hepatic shunting. In this setting, our initial experience with Infinia-Hawkeye -4 opens the possibility of developing individualised IRT based on patient-specific attenuation maps, especially for ${ }^{131} \mathrm{I}$ therapy of benign multinodular goiters and Graves' disease. Standardised uptake value scores (SUVs) dedicated to SPECT/CT imaging will also be useful for accurate semi-quantification of tracer uptake within normal and abnormal structures [28, $37,42,43]$. SUVs may be of interest in clinical routine and research for complementing the qualitative interpretation as demonstrated for Onco-PET and PET-CT with ${ }^{18}$ FDG [44]. Similarly, the introduction of CT-based AC allowed us to extend our pilot project to half-time MPI with attenuation correction, scatter correction, and resolution recovery (i.e. Cardiac for Evolution); thereby, suggesting the possibility of a future SPECT/CT MPI in only $5 \mathrm{~min}$.

The hardware fusion with low-dose SPECT/CT was complemented by an upgraded software package. This provided new tools for improving the data processing, and ultimately the interpretation confidence. This was particularly useful with MPI using AC applications, and bone studies using resolution recovery applications. A further step will be to develop multimodality applications and SPECT/CT-based radiation therapy.

In this project, we assessed a new low-dose multislice hybrid device before commercialisation on a large number of patients evaluated for various indications in routine clinical practice and research. Limitations in our study possibly relate to the short-term follow-up, the lack of systematical histopathological correlations in every single application, and the retrospective analyses. However, we emphasize the strategic relevance in clinical routine and research of an integrated SPECT/low-dose multislice CT in the context where PET/CT is firmly established in oncology, and also of volumetric CT increasingly used in cardiology. Table 15 summarizes the clinical relevance of SPECT/low-dose multislice CT system. The promising results obtained on Infinia-Hawkeye -4 require further confirmation through prospective studies. Well-designed studies are still needed to compare the performance of high-dose and/or highresolution CT versus multislice low-dose CT for integrated SPECT/CT devices.

\section{CONCLUSION}

The Infinia ${ }^{\mathrm{TM}}$ Hawkeye $\mathrm{e}^{\mathrm{TM}} 4$ slice, a new integrated SPECT/low-dose multislice CT was successfully implemented in routine clinical practice. As expected, the sub-optimal quality of the low-dose CT cannot displace the performance of a fully-diagnostic CT, but it did improve the interpretation of nuclear medicine images over routine planar imaging and SPECT alone. The low-end CT contributes to accurate anatomic localisation. Its utility for attenuation correction in cardiac and non-cardiac studies was also documented. This SPECT/CT model was found useful in experimental and clinical research, especially for 
development of new reconstruction algorithms extended to resolution recovery, (semi)quantification of tracer activity, and accurate dosimetry. Further evaluation is needed to confirm these preliminary conclusions.

\section{ACKNOWLEDGEMENTS}

The technical and clinical evaluation of the Infinia ${ }^{\mathrm{TM}}$ Hawkeye $^{\text {TM }} 4$ at South Street Hospital (London, Ontario, Canada) was part of the research agreement made between London Health Sciences Centre (LHSC) and GE Healthcare. Many thanks to GE application managers for their efficient collaboration during this project, especially Aharon Peretz, Reuven Brenner, Caroline Rochette, and Ken Efrati. Many thanks also go to the Nuclear Medicine technical staff from South Street Hospital for its valuable contribution to the implementation of the Infinia ${ }^{\mathrm{TM}}$ Hawkeye ${ }^{\mathrm{TM}} 4$, especially Bob Vanderwerf and Suzanne Quirk.

\section{CONFLICT OF INTEREST}

The authors disclose no conflict of interest with GE Healthcare and LHSC.

\section{ABBREVIATIONS}

\begin{tabular}{|c|c|c|}
\hline LHSC & $=$ & London Health Sciences Centre \\
\hline HWK -4 & $=$ & Infinia $^{\mathrm{TM}}$ Hawkeye $\mathrm{e}^{\mathrm{TM}} 4$ slice \\
\hline $\mathrm{PET} / \mathrm{CT}$ & $=$ & $\begin{array}{l}\text { Positron emission tomography/ } \\
\text { computed tomography }\end{array}$ \\
\hline SPECT/CT & $=$ & $\begin{array}{l}\text { Single photon emission } \\
\text { tomography/computed } \\
\text { tomography }\end{array}$ \\
\hline SPECT & $=$ & $\begin{array}{l}\text { Single photon emission } \\
\text { tomography }\end{array}$ \\
\hline VCT & $=$ & Volumetric CT \\
\hline $\mathrm{CT}$ & $=$ & Computed tomography \\
\hline US & $=$ & Ultrasound \\
\hline FD-CT & $=$ & Fully-diagnostic CT \\
\hline MRI & $=$ & Magnetic resonance imaging \\
\hline HR-MRI & $=$ & High-resolution MRI \\
\hline HR-US & $=$ & High-resolution US \\
\hline MPI & $=$ & Myocardial perfusion imaging \\
\hline LVEF & $=$ & Left ventricle ejection fraction \\
\hline TID & $=$ & Transient ischemic dilatation \\
\hline IRT & $=$ & Internal radiation therapy \\
\hline SLN & $=$ & Sentinel lymph node \\
\hline DTC & $=$ & Differentiated thyroid cancer \\
\hline BTD & $=$ & Benign thyroid diseases \\
\hline NET & $=$ & Neuroendocrine tumour \\
\hline MEN & $=$ & Multiple endocrine neoplasia \\
\hline SPA & $=$ & Single parathyroid adenoma \\
\hline CAD & $=$ & Coronary artery disease \\
\hline $\mathrm{CD}$ & $=$ & Crohn's disease \\
\hline
\end{tabular}

\begin{tabular}{|c|c|c|}
\hline BMI & $=$ & Body mass index \\
\hline PSA & $=$ & Prostate specific antigen \\
\hline iPTH & $=$ & Intact parathormone \\
\hline $\mathrm{Ca}$ & $=$ & Serum calcium \\
\hline SUV & $=$ & Standardised uptake value \\
\hline${ }^{18} \mathrm{FDG}$ & $=$ & ${ }^{18}$ F-fluorodeoxyglucose \\
\hline${ }^{18} \mathrm{FLT}$ & $=$ & ${ }^{18}$ F-fluoro-L-thymidine \\
\hline${ }^{18} \mathrm{FCH}$ & $=$ & ${ }^{18}$ F-fluoro-methyl-choline \\
\hline${ }^{68}$ Ga-DOTA-NOC & $=$ & $\begin{array}{l}{ }^{68} \text { Ga-labelled }[1,4,7,10- \\
\text { tetraazacyclododecane-1, 4, 7, 10- } \\
\text { tetraacetic acid]-1-Nal3- } \\
\text { octreotide }\end{array}$ \\
\hline${ }^{68} \mathrm{Ga}$-DOTA-TOC & $=$ & $\begin{array}{l}{ }^{68} \text { Ga-labeled }[1,4,7,10- \\
\text { tetraazacyclododecane-N, N', N", } \\
\text { N"'-tetraacetic acid]-d-Phe(1)- } \\
\text { Tyr(3)-octreotide }\end{array}$ \\
\hline${ }^{18} \mathrm{~F}-\mathrm{FDOPA}$ & $=$ & $\begin{array}{l}\text { L-3,4-Dihydroxy-6-(18)F-fluoro- } \\
\text { phenyl-alanine }\end{array}$ \\
\hline${ }^{99 m}$ Tc-MDP & $=$ & $\begin{array}{l}{ }^{99 m} \text { Tc-labelled metylenediphos- } \\
\text { phonate }\end{array}$ \\
\hline${ }^{99 m}$ Tc-HMPAO-WBC & $=$ & $\begin{array}{l}{ }^{99 m} \text { Tc-HMPAO-labelled white } \\
\text { blood cells }\end{array}$ \\
\hline${ }^{99 \mathrm{~m}} \mathrm{Tc}-\mathrm{RBC}$ & $=$ & ${ }^{99 \mathrm{~m}}$ Tc-labelled red blood cells \\
\hline${ }^{131 / 123} \mathrm{I}-\mathrm{MIBG}$ & $=$ & $\begin{array}{l}{ }^{131 / 123} \text { I-labelled metaiodobenzyl- } \\
\text { guanidine }\end{array}$ \\
\hline${ }^{99 m}$ Tc-MIBI & $=$ & $\begin{array}{l}{ }^{99 m} \text { Tc-labelled metoxyisobutyli- } \\
\text { sonitrile }\end{array}$ \\
\hline${ }^{201} \mathrm{Tl}$ & $=$ & ${ }^{201}$ Thallium \\
\hline${ }^{131} \mathrm{I}$ & $=$ & ${ }^{131}$ I-iodine \\
\hline${ }^{123} \mathrm{I}$ & $=$ & ${ }^{123} \mathrm{I}$-iodine \\
\hline Octreoscan $^{\circledR}$ & $=$ & ${ }^{111}$ In-labelled octreotide \\
\hline $\operatorname{ProstaScint}^{\circledR}$ & $=$ & ${ }^{111}$ In-labelled capromab pendetide \\
\hline $\operatorname{Bexxar}^{\circledR}$ & $=$ & ${ }^{131}$ I-labelled tositumomab \\
\hline Thyrogen $^{\circledR}$ & $=$ & $\begin{array}{l}\text { Recombinant human thyrostim- } \\
\text { ulin or rh-TSH }\end{array}$ \\
\hline TRT & $=$ & Treatment \\
\hline HT & $=$ & Hormone therapy \\
\hline pXRT & $=$ & Pelvic external radiation therapy \\
\hline SR & $=$ & Suspicion of recurrence \\
\hline IS & $=$ & Initial staging \\
\hline SW & $=$ & Salvage therapy work-up \\
\hline NED & $=$ & No evidence of disease \\
\hline PLD & $=$ & Pelvic local disease \\
\hline EPDD & $=$ & Extra-pelvic distant disease \\
\hline FOV & $=$ & Field of view \\
\hline WBS & $=$ & Whole-body scan \\
\hline PWB & $=$ & Planar whole-body \\
\hline
\end{tabular}




\begin{tabular}{|c|c|c|}
\hline $\mathrm{AC}$ & $=$ & Attenuation correction \\
\hline ACQC & $=$ & $\begin{array}{l}\text { Attenuation correction quality } \\
\text { control }\end{array}$ \\
\hline FBP & $=$ & Filtered back projection \\
\hline OSEM & $=$ & $\begin{array}{l}\text { Ordered subset expectation } \\
\text { maximisation }\end{array}$ \\
\hline IRAC & $=$ & $\begin{array}{l}\text { Iterative reconstruction with } \\
\text { attenuation correction }\end{array}$ \\
\hline IRNC & $=$ & $\begin{array}{l}\text { Iterative reconstruction without } \\
\text { attenuation correction }\end{array}$ \\
\hline HTRR & $=$ & Half-time resolution recovery \\
\hline $3 \mathrm{D}$ & $=$ & Three dimensions \\
\hline $4 \mathrm{D}$ & $=$ & $3 \mathrm{D}+$ time \\
\hline Oral C & $=$ & Oral contrast \\
\hline IV C & $=$ & Intravenous contrast \\
\hline GallB & $=$ & Gallbladder \\
\hline $\mathrm{L} \operatorname{Tr}$ & $=$ & Left truncation \\
\hline $\mathrm{R} \operatorname{Tr}$ & $=$ & Right truncation \\
\hline $\mathrm{Sm} \mathrm{Br}$ & $=$ & Small breast \\
\hline $\mathrm{Lg} \mathrm{Br}$ & $=$ & Large breast \\
\hline SW & $=$ & Software \\
\hline IQ & $=$ & Image quality \\
\hline
\end{tabular}

\section{REFERENCES}

[1] Townsend DW, Beyer T. A combined PET-CT scanner: the path to true image fusion. Br J Radiol 2002; 75: S24-S30.

[2] Schillaci O. Hybrid SPECT/CT: a new era for SPECT imaging? Eur J Nucl Med Mol Imaging 2005; 32(5): 521-4.

[3] Maurer AH. Combined imaging modalities: PET/CT and SPECT/CT. Health Phys 2008; 95(5): 571-6.

[4] Israel O, Mor M, Gaitini D, et al. Combined functional and structural evaluation of cancer patients with a hybrid camera-based PET/CT system using (18)F-FDG. J Nucl Med 2002; 43(9): 112936.

[5] Schillaci O, Danieli R, Manni C, Simonetti G. Is SPECT/CT with a hybrid camera useful to improve scintigraphic imaging interpretation? Nucl Med Commun 2004; 25(7): 705-10.

[6] Townsend DW. Multimodality imaging of structure and function. Phys Med Biol 2008; 53(4): R1-R39.

[7] van Dalen JA, Vogel WV, Corstens FH, Oyen WJ. Multi-modality nuclear medicine imaging: artefacts, pitfalls and recommendations. Cancer Imag 2007; 7(1): 77-83.

[8] Schepis T, Gaemperli O, Koepfli P, et al. Use of coronary calcium score scans from stand-alone multislice computed tomography for attenuation correction of myocardial perfusion SPECT. Eur J Nucl Med Mol Imag 2007; 34(1): 11-9.

[9] von Schulthess GK. Integrated modality imaging with PET-CT and SPECT-CT: CT issues. Eur Radiol 2005; 15(Suppl 4): 121-6..

[10] Couturier O, Luxen A, Chatal JF, Vuillez JP, Rigo P, Hustinx R. Fluorinated tracers for imaging cancer with positron emission tomography. Eur J Nucl Med Mol Imag 2004; 31(8): 1182-206.

[11] Vallabhajosula S. (18)F-labeled positron emission tomographic radiopharmaceuticals in oncology: an overview of radiochemistry and mechanisms of tumor localization. Semin Nucl Med 2007; 37(6): 400-19.
Antunes P, Ginj M, Zhang $\mathrm{H}$, et al. Are radiogallium-labelled DOTA-conjugated somatostatin analogues superior to those labelled with other radiometals? Eur J Nucl Med Mol Imag 2007; 34(7): 982-93.

[13] Belhocine T, Pierard G, De Labrassinne M, Lahaye T, Rigo P. Staging of regional nodes in AJCC stage I and II melanoma: 18FDG PET imaging versus sentinel node detection. Oncologist 2002; 7(4): 2718.

[14] Lind P, Kohlfürst S. Respective roles of thyroglobulin, radioiodine imaging, and positron emission tomography in the assessment of thyroid cancer. Semin Nucl Med 2006; 36(3): 194- 205.

[15] Lawrentschuk N, Davis ID, Bolton DM, Scott AM. Positron emission tomography and molecular imaging of the prostate: an update. BJU Int 2006; 97(5) : 923-31.

[16] Adams S, Baum R, Rink T, Schumm-Dräger PM, Usadel KH, Hör G. Limited value of fluorine-18 fluorodeoxyglucose positron emission tomography for the imaging of neuroendocrine tumours. Eur J Nucl Med 1998; 25(1): 79-83.

[17] Hendel RC, Corbett JR, Cullom SJ, DePuey EG, Garcia EV, Bateman TM. The value and practice of attenuation correction for myocardial perfusion SPECT imaging: A joint position statement from the American Society of Nuclear Cardiology and the Society of Nuclear Medicine. J Nucl Cardiol 2002; 9(1): 135-43.

[18] Patton JA, Slomka PJ, Germano G, Berman DS. Recent technologic advances in nuclear cardiology. J Nucl Cardiol 2007; 14(4): 501-13.

[19] O'connor MK, Kemp B, Anstett F, et al. A multicenter evaluation of commercial attenuation compensation techniques in cardiac SPECT using phantom models. J Nucl Cardiol 2002; 9(4): 361-76.

[20] Delbeke D, Coleman RE, Guiberteau MJ, et al. Society of Nuclear Medicine (SNM). Procedure Guideline for SPECT/CT Imaging 1.0. J Nucl Med 2006; 47(7) : 1227-34.

[21] Bischof Delaloye A, Carrió I, Cuocolo A, et al. White paper of the European Association of Nuclear Medicine (EANM) and the European Society of Radiology (ESR) on multimodality imaging. Eur J Nucl Med Mol Imag 2007; 34(8): 1147-51.

[22] Tagliabue L, Schillaci O. SPECT/CT in oncology: the fusion of two imaging modalities is a new standard of care. Q J Nucl Med Mol Imag 2007; 51(4): 285-9.

[23] Even-Sapir E. Imaging of malignant bone involvement by morphologic, scintigraphic, and hybrid modalities. J Nucl Med 2005; 46(8): 1356-67.

[24] Belhocine TZ, Scott AM, Even-Sapir E, Urbain JL, Essner R. Role of nuclear medicine in the management of cutaneous malignant melanoma. J Nucl Med 2006; 47(6): 957-67.

[25] Even-Sapir E, Flusser G, Lerman H, Lievshitz G, Metser U. SPECT/multislice low-dose CT: a clinically relevant constituent in the imaging algorithm of nononcologic patients referred for bone scintigraphy. J Nucl Med 2007; 48(2): 319-24.

[26] Shah C, Patton JA, Sandler MP. How much CT is needed in nuclear medicine. Eur J Nucl Med Mol Imag 2008; 35(10): 175960 .

[27] Berman DS, Hachamovitch R, Shaw LJ, et al. Roles of nuclear cardiology, cardiac computed tomography, and cardiac magnetic resonance: assessment of patients with suspected coronary artery disease. J Nucl Med 2006; 47(7): 74-82.

[28] Thierens HM, Monsieurs MA, Bacher K. Patient dosimetry in radionuclide therapy: the whys and the wherefores. Nucl Med Commun 2005; 26(7): 593-9.

[29] Roach PJ, Schembri GP, Ho Shon IA, Bailey EA, Bailey DL SPECT/CT imaging using a spiral CT scanner for anatomical localization: Impact on diagnostic accuracy and reporter confidence in clinical practice. Nucl Med Commun 2006; 27(12): 977-87.

[30] Keidar Z, Israel O, Krausz Y. SPECT/CT in tumor imaging: technical aspects and clinical applications. Semin Nucl Med 2003; 33(3): 205-18.

[31] O'Connor MK, Kemp BJ. Single-photon emission computed tomography/computed tomography: basic instrumentation and innovations. Semin Nucl Med 2006; 36(4): 258-66.

[32] The weekly source of radiology professionals. SPECT/CT Systems. Image. 2008; 21.

[33] Jacene HA, Goetze S, Patel H, Wahl RL, Ziessman HA. Advantages of Hybrid SPECT/CT vs SPECT Alone. Open Med Imag J 2008; 13(2): 67-79.

[34] Bailey DL, Roach PJ, Bailey EA, Hewlett J, Keijzers R. Development of a cost-effective modular SPECT/CT scanner. Eur J Nucl Med Mol Imag 2007; 34(9): 1415-26. 
[35] Buck AK, Nekolla S, Ziegler S, et al. SPECT/CT. J Nucl Med 2008; 49(8): 1305-19.

[36] Chowdhury FU, Scarsbrook AF. The role of hybrid SPECT-CT in oncology: current and emerging clinical applications. Clin Radiol 2008; 63(3): 241-51.

[37] Bybel B, Brunken RC, DiFilippo FP, Neumann DR, Wu G, Cerqueira MD. SPECT/CT imaging: clinical utility of an emerging technology. Radiographics 2008; 28(4): 1097-113.

[38] Hamann M, Aldridge M, Dickson J, Endozo R, Lozhkin K, Hutton BF. Evaluation of a low-dose/slow-rotating SPECT-CT system. Phys Med Biol 2008; 53(10): 2495-508.

[39] Belhocine T, Akincioglu C, Gambhir S, et al. Evaluation of attenuation correction in myocardial perfusion imaging on InfiniaHawkeye-4 slice (GE Healthcare). J Nucl Cardiol 2007; 14(2): S40.
[40] van der Ploeg IM, Valdés Olmos RA, Kroon BB, Nieweg OE. The Hybrid SPECT/CT as an Additional Lymphatic Mapping Tool in Patients with Breast Cancer. World J Surg 2008; 32(9): 1930-4.

[41] Valdés Olmos RA, Vidal-Sicart S, Nieweg OE. SPECT-CT and realtime intraoperative: new tools for sentinel node localization and radioguided surgery? Eur J Nucl Med Mol Imag 2008 Oct 18. [Epub ahead of print].

[42] Frey EC. SPECT/CT for Dosimetry Calculations. Hybrid SPECT/CT Imaging in Clinical Practice. Israel O, Goldsmith SJ, Eds. Informa HealthCare. Chap. 10: 157-180.

[43] Shcherbinin S, Celler A, Belhocine T, Vanderwerf R, Driedger A. Accuracy of quantitative reconstructions in SPECT/CT imaging. Phys Med Biol 2008; 53(17): 4595-604.

[44] Marsden PK. Quantification in PET: what is it? Can we do it? Do we need it? Nucl Med Commun 2004; 25(7): 635-6.

(C) Belhocine et al.; Licensee Bentham Open.

This is an open access article licensed under the terms of the Creative Commons Attribution Non-Commercial License (http://creativecommons.org/licenses/by$\mathrm{nc} / 3.0 /$ ) which permits unrestricted, non-commercial use, distribution and reproduction in any medium, provided the work is properly cited. 